\title{
Slip Flow in Porous Medium of Micropolar Fluid in a Rectangular Microchannel under the Effect of a Magnetic Field
}

\section{Agoor B.M.*}

Faculty of Science, Department of Mathematics, Fayoum University, Fayoum, Egypt

\begin{abstract}
The magnetohydrodynamic flow of micropolar fluid through porous medium inside the rectangular microchannel is investigated. The flow is subjected to a uniform magnetic field. This phenomenon is modulated mathematically by a system of linear differential equations which describe the continuity, momentum and angular momentum equations of the considered fluid. The system of differential equations has been solved analytically. We have obtained the velocity and microrotation vectors in terms of a Fourier series. The volumetric flow rate is calculated and the effect of various parameters like medium permeability, magnetic field, the Knudsen number, the microrotation parameter and coupling parameter are discussed and illustrated graphically through a set of figures.
\end{abstract}

Keywords: Micropolar fluid; Porous medium; Slip-flow; Microrotation vector; Rectangular microchannel

\section{Introduction}

In nature and various engineering fields, there are a large number of fluids, containing small particles, such as blood, polymeric suspensions, colloids, liquid crystals, and lubricants. Their bulk fluid properties are different from those of Newtonian fluids, due to fluidparticle interaction and particle rotation on the bulk flow. Eringen [1] constructed the micropolar fluid model and gave the analytical solution for Poiseuille flow in a pipe. The micropolar fluid model has been extensively used. Hogan [2] adopted the model to investigate the blood flow in an idealized stenosis. The flow through a tube of uniform cross section is attractive for researchers since different types of cross sections will involve different types of solutions. In a very popular paper, Sparrow [3] has considered the entrance flow of a viscous fluid through a tube of arbitrary cross section. This problem was analyzed numerically in the case of a rectangular dust using finite element method by Comini and Chien [4,5] studied the unsteady flow of a viscous fluid through a rectangular channel. This problem was extended by Gupta Prem [6] to study the nature of flow through a rectangular tube filled with the saturated porous bed. Various types of unsteady flows of a secondgrade fluid though a duct of the uniform crosses section were discussed by Emin Erdogan [7]. But very few papers are available in the case of micropolar fluid flow through the rectangular channel under constant and periodic pressure gradients. The steady flow of an incompressible conducting micropolar fluid through a rectangular channel with a uniform cross-section in the presence of a transverse magnetic field with suction and injection at the side walls was considered by Ramana Murthy [8]. The slip-flow of a Newtonian fluid past a linearly stretching sheet was considered Andersson [9]. The partial slip was controlled by a dimensionless slip factor, which varies between zero (total adhesion) and infinity (full slip). Misra [10] studied blood flow through a stenosed arterial segment by taking into account the slip velocity at the wall of the artery. Consideration of the Non-Newtonian character of blood was made, where a constitutive relation of blood was described by Hershel-Bulkley equation. The effect of slip at the arterial wall in the presence of mild, moderate, and severe stenosis growth at the lumen of an artery was investigated. Lok [11] considered the problem of steady two-dimensional boundary layer flow of a micropolar fluid near an oblique stagnation point on a fixed surface with Navier's slip condition. It was found that the flow characteristics depend strongly on the micropolar and slip parameters. Villevo Adanhounme [12] investigated the Navier stokes flow equations of micropolar fluids by peristaltic pumping through the cylindrical tube, taking into account the slip boundary conditions at the wall and using the suitable change of variables. Shangjun Ye [13] Studied laminar flow of micropolar fluid in rectangular microchannels. The governing equations solved numerically to obtained the velocity profiles and microrotation gyrations by a procedure based on the Chebyshev collocation method. Vimala [14] studied the laminar two-dimensional flow of a micropolar fluid through a porous channel with variable permeability. The electroosmotic flow of a micropolar bio-fluid in a microchannel under the action of an alternating electric field was described by Misra [15]. In this paper, we investigated the slip flow in a porous medium of EyringPowell micropolar fluid in a rectangular microchannel under the effect of a magnetic field. The rest of this paper is organized as follows: in the next section we present the details of the models we analyzed, and then we use Fourier series for solving the equations. In the results and discussions, we summarize our results.

\section{Formulation of the Problem}

The geometry of the problem is shown in Figure 1. The center of the coordinate system is located at the bottom left corner of the channel. The dimensions of the channel in $\mathrm{y}$ and $\mathrm{z}$ directions are $\mathrm{a}$ and $\mathrm{b}$ respectively and $\mathrm{x}$-coordinate is measured along the axis of the channel.

The equations of steady flow in porous medium for an incompressible conducting Eyring-Powell micropolar fluid in the presence of a magnetic field as described by Eringen [16] are given by:

$$
\underline{\nabla} \cdot \underline{q}=0
$$

*Corresponding author: Agoor BM, Faculty of Science, Department of Mathematics, Fayoum University, Fayoum, Egypt, Tel: 084-6371482; E-mail: bma00@fayoum.edu.eg

Received November 20, 2017; Accepted December 11, 2017; Published January 14, 2018

Citation: Agoor BM (2018) Slip Flow in Porous Medium of Micropolar Fluid in a Rectangular Microchannel under the Effect of a Magnetic Field. J Appl Mech Eng 7: 298. doi:10.4172/2168-9873.1000298

Copyright: ( 2018 Agoor BM. This is an open-access article distributed under the terms of the Creative Commons Attribution License, which permits unrestricted use, distribution, and reproduction in any medium, provided the original author and source are credited. 


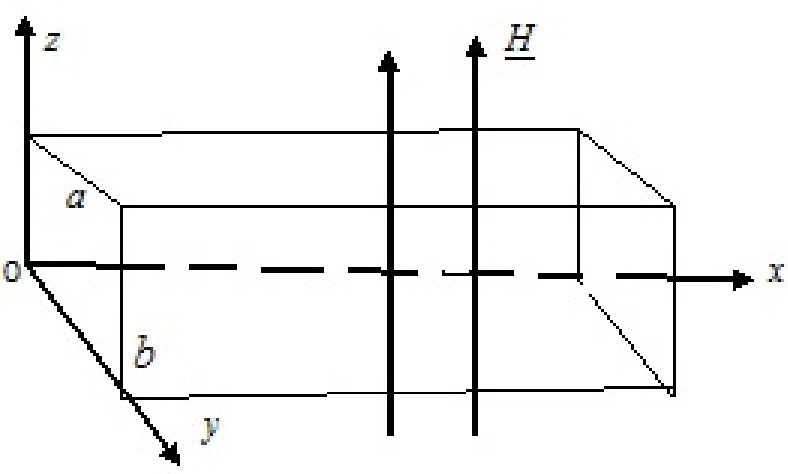

Figure 1: The schematic of the micro-channel.

$$
\rho(\underline{q} \cdot \underline{\nabla}) \underline{q}=-\underline{\nabla} p+k(\underline{\nabla} \wedge \underline{N})-(\mu+k)(\underline{\nabla} \wedge \underline{\nabla} \wedge \underline{q})-\frac{\mu}{k_{p}} \underline{q}+\underline{J} \wedge \underline{H}+\underline{\nabla} \cdot \underline{\tau}
$$

And

$$
\rho j(\underline{q} \cdot \underline{\nabla}) \underline{N}=-2 k \underline{N}+k(\underline{\nabla} \wedge \underline{q})-\gamma(\underline{\nabla} \wedge \underline{\nabla} \wedge \underline{N})+
$$

$$
(\alpha+\beta+\gamma) \underline{\nabla}(\underline{\nabla} \cdot \underline{N})(3)
$$

Where $\rho, \underline{q}, p, \underline{N}$ and $\underline{J}$ are the density, the velocity vector, pressure, the microrotation vector and the magnetic field, $k$ is the cross viscosity, $\mu$ is the dynamic viscosity of biofluid, $k_{p}$ is the permeability of porous medium. $J$ is the current density and $j$ is the micro-gyration parameter, and $\tau_{i j}$ is the stress tensor for an Eyring-Powell fluid which describes the shear behavior of a number of viscoelastic fluids including polymers, biofluids etc, as discussed earlier, this takes the following form

$$
\tau_{i j}=\mu \frac{\partial q_{i}}{\partial x_{j}}+\frac{1}{\beta^{*}} \sinh ^{-1}\left(\frac{1}{c} \frac{\partial q_{i}}{\partial x_{j}}\right)
$$

Where $\beta^{\star}$ and $c$ are the Eyring-Powell material characteristics, we can simplify $\tau_{i j}$ by taking first approximations of the hyperbolic functions as

$$
\tau_{i j}=\left(\mu+\frac{1}{\beta^{*} c}\right) \frac{\partial q_{i}}{\partial x_{j}}, \quad\left|\frac{1}{c} \frac{\partial q_{i}}{\partial x_{j}}\right|\langle 1
$$

The material coefficients $\alpha, \beta$, $\gamma$ related by the inequalities

$$
2 \mu+k \geq 0, k \geq 0,3 \alpha+\beta+\gamma \geq 0, \gamma \geq|\beta|
$$

The velocity field, microrotation vector, and magnetic field are defined as

$$
\left.\begin{array}{l}
\underline{q}=u(y, z) \underline{i}, \underline{N}=(0, A, B), \underline{H}=H_{0} \underline{k} \\
\underline{J}=\sigma(\underline{E}+\underline{q} \wedge \underline{H}), A=A(y, z), B=B(y, z)
\end{array}\right\}
$$

Where $A$ and $B$ are the component of microrotation vector, $\underline{E}$ is the electrical field and $\sigma$ is the electrical conductivity. We can neglect the electrical field $\underline{H}$ compare with the magnetic field $\underline{H}$. We assume that the electrical field $\underline{H}$ and the induced magnetic field due to the motion of the fluid do not disturb the applied magnetic field. This assumption is reasonable if the magnetic Reynolds number is very small. Hence, $\underline{H}$ can be taken as a constant applied magnetic field $H_{0} \underline{k}$. substitute from equation (5) and (6) in equations (2) and (3) we obtain,

$$
\begin{aligned}
& 0=-\frac{\partial P}{\partial x}+k\left(\frac{\partial B}{\partial y}-\frac{\partial A}{\partial z}\right)+\left(2 \mu+k+\frac{1}{\beta^{*} c}\right)\left(\frac{\partial^{2} u}{\partial y^{2}}+\frac{\partial^{2} u}{\partial z^{2}}\right)-\frac{\mu}{k_{p}} u-\sigma u H_{0}^{2} \\
& 0=-2 k A+k \frac{\partial u}{\partial z}+\gamma \frac{\partial}{\partial z}\left(\frac{\partial A}{\partial z}-\frac{\partial B}{\partial y}\right)+(\alpha+\beta+\gamma) \frac{\partial}{\partial y}\left(\frac{\partial A}{\partial y}+\frac{\partial B}{\partial z}\right) \\
& 0=-2 k B-k \frac{\partial u}{\partial y}-\gamma \frac{\partial}{\partial y}\left(\frac{\partial A}{\partial z}-\frac{\partial B}{\partial y}\right)+(\alpha+\beta+\gamma) \frac{\partial}{\partial z}\left(\frac{\partial A}{\partial y}+\frac{\partial B}{\partial z}\right)
\end{aligned}
$$

In the present study, slip-flow in rectangular microchannel is investigated under the boundary conditions for slip-flow regime, the hydrodynamic boundary conditions are:

$$
\begin{aligned}
& \left.\begin{array}{l}
u=u_{s}, \underline{N}=(0, A, B)=\underline{0} \text { at } y=0 \text { and } z=0 \\
u=u_{s}, \underline{N}=(0, A, B)=\underline{0} \text { at } y=a \text { and } z=b
\end{array}\right\} \\
& u_{s}=\left(\frac{2-F_{v}}{F_{v}}\right) \lambda\left(\frac{\partial u}{\partial n}\right)_{w}
\end{aligned}
$$

Where $u_{s}$ is the slip velocity at the walls Eckert and Goniak $[17,18]$, and $F_{v}$ is the tangential momentum accommodation coefficient, $\lambda$ is the molecular mean free path and $\left(\frac{\partial u}{\partial n}\right)_{w}$ is the velocity gradients normal to the wall. To facilitate solutions, we now introduce a set of transformations which render the conservation equations dimensionless, defining:

$$
\begin{aligned}
& y=a y^{\prime}, z=a z^{\prime}, x=a x^{\prime}, u=u_{m} u^{\prime}, A=\frac{u_{m}}{a} A^{\prime}, B=\frac{u_{m}}{a} B^{\prime}, \frac{\partial p}{\partial x}=\frac{u_{m}^{2}}{a} p_{0}, \\
& \delta=\left(\frac{\gamma}{\alpha+\beta+\gamma}\right), h=\frac{b}{a}, R_{e}=\frac{a u_{m}}{\left(2 \mu+k+\beta^{*} c\right)}, c_{0}=\frac{k}{\left(2 \mu+k+\beta^{*} c\right)}, s=\frac{k a^{2}}{\gamma}, \\
& M^{2}=\frac{\sigma H_{0}^{2} a^{2}}{\left(2 \mu+k+\beta^{*} c\right)}, \frac{1}{k_{p}^{*}}=\frac{\mu a^{2}}{k_{p}\left(2 \mu+k+\beta^{*} c\right)}, G=\frac{\partial B^{\prime}}{\partial y^{\prime}}-\frac{\partial A^{\prime}}{\partial z^{\prime}}, F=\frac{\partial A^{\prime}}{\partial y^{\prime}}+\frac{\partial B^{\prime}}{\partial z^{\prime}}, \\
& \beta_{v}=\frac{2-F_{v}}{F_{v}}, k_{m}=\frac{\lambda}{a}, L=2 s R_{e} p_{0}
\end{aligned}
$$

Where $C_{0}$ is coupling parameter, $s$ and $\delta$ are couple stress parameters, $R_{e}$ is the Reynolds number, $k_{m}$ is the Knudsen number and $k_{p}^{*}$ is the permeability of porous medium, implementing these expressions and proceeding with the analysis, the governing equations (7)-(9) now reduce to the following partial differential equations in terms of the dependent variable, dimensionless form after dropping dash mark are given by:

$$
\begin{aligned}
& -R_{e} p_{0}+c_{0} G+\nabla^{2} u-\frac{1}{k_{p}^{*}} u-M^{2} u=0 \\
& -2 s A+s \frac{\partial u}{\partial z}-\frac{\partial G}{\partial z}+\frac{1}{\delta} \frac{\partial F}{\partial y}=0 \\
& -2 s B-s \frac{\partial u}{\partial y}+\frac{\partial G}{\partial y}+\frac{1}{\delta} \frac{\partial F}{\partial z}=0
\end{aligned}
$$

The corresponding transformed boundary conditions are now given by:

$$
\left.\begin{array}{l}
u=u_{s}, \underline{N}=(0, A, B)=\underline{0} \text { at } y=0 \text { and } z=0 \\
u=u_{s}, \underline{N}=(0, A, B)=\underline{0} \text { at } y=1 \text { and } z=h \\
u_{s}=\beta_{v} k_{m}\left(\frac{\partial u}{\partial n}\right)_{w}
\end{array}\right\}
$$


Eliminating $A, B$ and $F$ from (14) and (15), we get the equation of $G$ as:

$$
\left(\nabla^{2}-2 s\right) G=s \nabla^{2} u
$$

Eliminating $A, B$ and $G$ from (14) and (15), we get the equation of $F$ as:

$$
\left(\nabla^{2}-2 s \delta\right) F=0
$$

Use equation (17) to eliminate $G$ from equation (13) we get,

$$
\nabla^{4} u-\left(2 s+M^{2}+\frac{1}{k_{p}^{*}}-c_{0} s\right) \nabla^{2} u+\left(\frac{2 s}{k_{p}^{*}}+2 s M^{2}\right)=-L
$$

To solve equation (19) under slip boundary conditions (16), we employ $u(y, z)$ in the form of

$$
u(y, z)=\sum_{n=0}^{\infty} u_{n}(y) \cos \left(t_{n} z\right), t_{n}=\frac{(2 n+1) \pi}{2 h}
$$

$u_{n}$ is obtained by substituting (20) in (19) and using Fourier series for RHS of (19), we get,

$$
\frac{d^{4} u_{n}(y)}{d y^{4}}-a_{1} \frac{d^{2} u_{n}(y)}{d y^{2}}+a_{2} u_{n}(y)=\frac{2 l(-1)^{n+1}}{t_{n} h}
$$

Solution of equation (21) can be taken as:

$$
u_{n}(y)=c_{1} e^{-\alpha y}+c_{2} e^{\alpha y}+c_{3} e^{-\alpha y}+c_{4} e^{\alpha y}-\frac{2(-1)^{n} L}{h a_{2} t_{n}}
$$

Where

$$
\left.\begin{array}{l}
\alpha_{1}=\frac{\sqrt{a_{1}-\sqrt{a_{1}^{2}-4 a_{2}}}}{\sqrt{2}}, \alpha_{2}=\frac{\sqrt{a_{1}+\sqrt{a_{1}^{2}-4 a_{2}}}}{\sqrt{2}}, \\
a_{1}=2 s+M^{2}+\frac{1}{k_{p}^{*}}+t_{n}^{2}-c_{0} s, \\
a_{2}=t_{n}^{4}+t_{n}^{2}\left(2 s+M^{2}+\frac{1}{k_{p}^{*}}-c_{0} s\right)+\left(\frac{2 s}{k_{p}^{*}}+2 s M^{2}\right)
\end{array}\right\}
$$

For solving equation (18), we let:

$$
F(y, z)=\sum_{n=0}^{\infty} F_{n}(y) \sin \left(t_{n} z\right)
$$

Substitute (24) in (18) we obtain:

$$
\frac{d^{2} F_{n}(y)}{d y^{2}}-\left(2 s \delta+t_{n}^{2}\right) F_{n}=0
$$

Hence solution of $F_{n}$ is written as:

$$
F_{n}(y)=c_{5} e^{-\beta_{1} y}+c_{6} e^{\beta_{1} y}, \quad \beta_{1}=\sqrt{2 s \delta+t_{n}^{2}}
$$

Substituting (20) in (13), we have:

$$
G(y, z)=\left(\frac{R_{e} p_{0}}{c_{0}}\right)+\sum_{n=0}^{\infty}\left[\left(\frac{-1}{c_{0}}\right) \frac{d^{2} u_{n}(y)}{d y^{2}}+\left(\frac{t_{n}^{2}}{c_{0}}+\frac{M^{2}}{c_{0}}+\frac{1}{c_{0} k_{p}^{*}}\right) u_{n}(y)\right] \cos \left(t_{n} z\right)
$$

To match with the expressions for $F$ in (24) and $G$ in (27), the solution for $A$ and $B$ can be taken in the form:

$$
A(y, z)=\sum_{n=0}^{\infty} A_{n}(y) \sin \left(t_{n} z\right), B(y, z)=\sum_{n=0}^{\infty} B_{n}(y) \cos \left(t_{n} z\right)
$$

Substituting (20), (26), (27) and (28) in (14) and (15) we get:

$$
\begin{gathered}
A_{n}(y)=\left(\frac{-t_{n}}{2 s c_{0}}\right)\left[\alpha_{1}^{2} c_{1} e^{-\alpha y}+\alpha_{1}^{2} c_{2} e^{\alpha y}+\alpha_{2}^{2} c_{3} e^{-\alpha y}+\alpha_{2}^{2} c_{4} e^{\alpha y}\right]+ \\
{\left[\left(\frac{-t_{n}}{2}+\frac{t_{n}^{3}}{2 s c_{0}}+\frac{t_{n} M^{2}}{2 s c_{0}}+\frac{t_{n}}{2 s c_{0} k_{p}^{*}}\right)\left(c_{1} e^{-\alpha y}+c_{2} e^{\alpha y}+c_{3} e^{-\alpha y}+c_{4} e^{\alpha y}-\left(\frac{2(-1)^{n} L}{h a_{2} t_{n}}\right)\right)\right]} \\
-\left(\frac{\beta_{1}}{2 s \delta}\right) c_{5} e^{-\beta y}-\left(\frac{\beta_{1}}{2 s \delta}\right) c_{6} e^{\beta y}
\end{gathered}
$$

And

$$
\begin{array}{r}
B_{n}(y)=\left(\frac{-1}{2}\right)\left[-\alpha_{1} c_{1} e^{-\alpha y}+\alpha_{1} c_{2} e^{\alpha y}-\alpha_{2} c_{3} e^{-\alpha y}+\alpha_{2} c_{4} e^{\alpha y}\right] \\
-\left(\frac{1}{2 s c_{0}}\right)\left[-\alpha_{1}^{3} c_{1} e^{-\alpha y}+\alpha_{1}^{3} c_{2} e^{\alpha y}-\alpha_{2}^{3} c_{3} e^{-\alpha y}+\alpha_{2}^{3} c_{4} e^{\alpha y}\right]+ \\
\left(\frac{t_{n}^{2}}{2 s c_{0}}+\frac{M^{2}}{2 s c_{0}}+\frac{1}{2 s c_{0} k_{p}^{*}}\right)\left(-\alpha_{1} c_{1} e^{-\alpha y}+\alpha_{1} c_{2} e^{\alpha y}-\alpha_{2} c_{3} e^{-\alpha y}+\alpha_{2} c_{4} e^{\alpha y}\right) \\
+\left(\frac{t_{n}}{2 s \delta}\right)\left[c_{5} e^{-\beta y}+c_{6} e^{\beta y}\right](30)
\end{array}
$$

Using boundary conditions (16) on $u$ i.e., $u=u_{s}$ at $y=0$ and $y=1$, we get:

$$
c_{1}+c_{2}+c_{3}+c_{4}-\left(\frac{2(-1)^{n} L}{h a_{2} t_{n}}\right)=\beta_{v} k_{m}\left[-\alpha_{1} c_{1}+\alpha_{1} c_{2}-\alpha_{2} c_{3}+\alpha_{2} c_{4}\right]
$$

And

$$
\begin{aligned}
c_{1} e^{-\alpha}+c_{2} e^{\alpha}+c_{3} e^{-\alpha}+c_{4} e^{\alpha}-\left(\frac{2(-1)^{n} L}{h a_{2} t_{n}}\right) \\
=\beta_{v} k_{m}\left[-\alpha_{1} c_{1} e^{-\alpha}+\alpha_{1} c_{2} e^{\alpha^{\alpha}}-\alpha_{2} c_{3} e^{-\alpha}+\alpha_{2} c_{4} e^{\alpha}\right]
\end{aligned}
$$

Using the condition on $A$ that $A=0$ at $y=0$ and $y=1$, we get:

$$
\begin{aligned}
0= & \left(\frac{-t_{n}}{2 s c_{0}}\right)\left[\alpha_{1}^{2} c_{1}+\alpha_{1}^{2} c_{2}+\alpha_{2}^{2} c_{3}+\alpha_{2}^{2} c_{4}\right]+ \\
& {\left[\frac{-t_{n}}{2}+\frac{t_{n}^{3}}{2 s c_{0}}+\frac{t_{n} M^{2}}{2 s c_{0}}+\frac{t_{n}}{2 s c_{0} k_{p}^{*}}\right]\left[c_{1}+c_{2}+c_{3}+c_{4}-\left(\frac{2(-1)^{n} L}{h a_{2} t_{n}}\right)\right]-} \\
& \left(\frac{\beta_{1}}{2 s \delta}\right)\left(c_{5}-c_{6}\right)
\end{aligned}
$$

And

$$
\begin{array}{r}
0=\left(\frac{-t_{n}}{2 s c_{0}}\right)\left[\alpha_{1}^{2} c_{1} e^{-\alpha}+\alpha_{1}^{2} c_{2} e^{\alpha}+\alpha_{2}^{2} c_{3} e^{-\alpha}+\alpha_{2}^{2} c_{4} e^{\alpha}\right]+ \\
{\left[\frac{-t_{n}}{2}+\frac{t_{n}^{3}}{2 s c_{0}}+\frac{t_{n} M^{2}}{2 s c_{0}}+\frac{t_{n}}{2 s c_{0} k_{p}^{*}}\right]\left[\begin{array}{l}
\left.c_{1} e^{-\alpha}+c_{2} e^{\alpha}+c_{3} e^{-\alpha}+c_{4} e^{\alpha}-\left(\frac{2(-1)^{n} L}{h a_{2} t_{n}}\right)\right]- \\
\left(\frac{\beta_{1}}{2 s \delta}\right)\left(c_{5} e^{-\beta}-c_{6} e^{\beta}\right)(34)
\end{array}\right.}
\end{array}
$$

Similarly, boundary conditions on $B$ that $B=0$ at $y=0$ and $y=1$, yields:

$$
\begin{aligned}
0=\left(\frac{-1}{2}\right)\left[-\alpha_{1} c_{1}+\alpha_{1} c_{2}-\alpha_{2} c_{3}+\alpha_{2} c_{4}\right] & \\
-\left(\frac{1}{2 s c_{0}}\right) & {\left[-\alpha_{1}^{3} c_{1}+\alpha_{1}^{3} c_{2}-\alpha_{2}^{3} c_{3}+\alpha_{2}^{3} c_{4}\right]+} \\
& \left(\frac{t_{n}^{2}}{2 s c_{0}}+\frac{M^{2}}{2 s c_{0}}+\frac{1}{2 s c_{0} k_{p}^{*}}\right)\left(-\alpha_{1} c_{1}+\alpha_{1} c_{2}-\alpha_{2} c_{3}+\alpha_{2} c_{4}\right) \\
& +\left(\frac{t_{n}}{2 s \delta}\right)\left[c_{5}+c_{6}\right]
\end{aligned}
$$

And 


$$
\begin{array}{r}
0=\left(\frac{-1}{2}\right)\left[-\alpha_{1} c_{1} e^{-\alpha}+\alpha_{1} c_{2} e^{\alpha}-\alpha_{2} c_{3} e^{-\alpha}+\alpha_{2} c_{4} e^{\alpha}\right] \\
-\left(\frac{1}{2 s c_{0}}\right)\left[-\alpha_{1}^{3} c_{1} e^{-\alpha}+\alpha_{1}^{3} c_{2} e^{\alpha}-\alpha_{2}^{3} c_{3} e^{-\alpha}+\alpha_{2}^{3} c_{4} e^{\alpha}\right]+ \\
\left(\frac{t_{n}^{2}}{2 s c_{0}}+\frac{M^{2}}{2 s c_{0}}+\frac{1}{2 s c_{0} k_{p}^{*}}\right)\left(-\alpha_{1} c_{1} e^{-\alpha}+\alpha_{1} c_{2} e^{\alpha}-\alpha_{2} c_{3} e^{-\alpha}+\alpha_{2} c_{4} e^{\alpha}\right) \\
+\left(\frac{t_{n}}{2 s \delta}\right)\left[c_{5} e^{-\beta}+c_{6} e^{\beta}\right](36)
\end{array}
$$

The equations from (31) to (36) are solved for find the constants $c_{1}$ $c_{2}, c_{3}, c_{4}, c_{5}$, and $c_{6}$, hence the solutions for $u, A$ and $B$ can be found.

\section{Volumetric flow rate}

The volumetric flow rate $Q$ in non-dimensional form is given by:

$$
\begin{aligned}
Q= & \int_{y=0}^{1} \int_{z=0}^{h} u(y, z) d z d y \\
Q= & \sum_{n=0}^{\infty} \frac{1}{t_{n}}\left\{\frac{-c_{1}}{\alpha_{1}} e^{-\alpha}+\frac{c_{2}}{\alpha_{1}} e^{\alpha}-\frac{c_{3}}{\alpha_{2}} e^{-\alpha}+\frac{c_{4}}{\alpha_{2}} e^{\alpha}\right. \\
& \left.-\left(\frac{2(-1)^{n} L}{h a_{2} t_{n}}\right)+\frac{c_{1}}{\alpha_{1}}-\frac{c_{2}}{\alpha_{1}}+\frac{c_{3}}{\alpha_{2}}-\frac{c_{4}}{\alpha_{2}}\right\} \sin \left(h t_{n}\right)
\end{aligned}
$$

Where

$c_{1}=\frac{\Delta_{1}}{\Delta}, c_{2}=\frac{\Delta_{2}}{\Delta}, c_{4}=\frac{\Delta_{4}}{\Delta}, c_{4}=\frac{\Delta_{4}}{\Delta}, c_{6}=\frac{\Delta_{6}}{\Delta}$ and $c_{6}=\frac{\Delta_{6}}{\Delta}$

And

$$
\Delta=\left|\begin{array}{cccccc}
\xi_{1} & \xi_{2} & \xi_{3} & \xi_{4} & 0 & 0 \\
\xi_{1} e^{-\alpha_{1}} & \xi_{2} e^{\alpha_{1}} & \xi_{3} e^{-\alpha_{2}} & \xi_{4} e^{\alpha_{2}} & 0 & 0 \\
\psi_{1} & \psi_{1} & \psi_{2} & \psi_{2} & -\xi_{8} & \xi_{8} \\
\psi_{1} e^{-\alpha_{1}} & \psi_{1} e^{\alpha_{1}} & \psi_{2} e^{-\alpha_{2}} & \psi_{2} e^{\alpha_{2}} & -\xi_{8} e^{-\beta_{1}} & \xi_{8} e^{\beta_{1}} \\
\psi_{3} & -\psi_{3} & \psi_{4} & -\psi_{4} & \left(\frac{t_{n}}{s \delta}\right) & \left(\frac{t_{n}}{s \delta}\right) \\
\psi_{3} e^{-\alpha_{1}} & -\psi_{3} e^{\alpha_{1}} & \psi_{4} e^{-\alpha_{2}} & -\psi_{4} e^{\alpha_{2}} & \left(\frac{t_{n}}{s \delta}\right) e^{-\beta_{1}} & \left(\frac{t_{n}}{s \delta}\right) e^{\beta_{1}}
\end{array}\right|,
$$

$$
\Delta_{1}=\left|\begin{array}{cccccc}
\xi_{5} & \xi_{2} & \xi_{3} & \xi_{4} & 0 & 0 \\
\xi_{5} & \xi_{2} e^{\alpha_{1}} & \xi_{3} e^{-\alpha_{2}} & \xi_{4} e^{\alpha_{2}} & 0 & 0 \\
\xi_{5} \xi_{7} & \psi_{1} & \psi_{2} & \psi_{2} & -\xi_{8} & \xi_{8} \\
\xi_{5} \xi_{7} & \psi_{1} e^{\alpha_{1}} & \psi_{2} e^{-\alpha_{2}} & \psi_{2} e^{\alpha_{2}} & -\xi_{8} e^{-\beta_{1}} & \xi_{8} e^{\beta_{1}} \\
0 & -\psi_{3} & \psi_{4} & -\psi_{4} & \left(\frac{t_{n}}{s \delta}\right) & \left(\frac{t_{n}}{s \delta}\right) \\
0 & -\psi_{3} e^{\alpha_{1}} & \psi_{4} e^{-\alpha_{2}} & -\psi_{4} e^{\alpha_{2}} & \left(\frac{t_{n}}{s \delta}\right) e^{-\beta_{1}} & \left(\frac{t_{n}}{s \delta}\right) e^{\beta_{1}}
\end{array}\right|,
$$$$
\Delta_{2}=\left|\begin{array}{cccccc}
\xi_{1} & \xi_{5} & \xi_{3} & \xi_{4} & 0 & 0 \\
\xi_{1} e^{-\alpha_{1}} & \xi_{5} & \xi_{3} e^{-\alpha_{2}} & \xi_{4} e^{\alpha_{2}} & 0 & 0 \\
\psi_{1} & \xi_{5} \xi_{7} & \psi_{2} & \psi_{2} & -\xi_{8} & \xi_{8} \\
\psi_{1} e^{-\alpha_{1}} & \xi_{5} \xi_{7} & \psi_{2} e^{-\alpha_{2}} & \psi_{2} e^{\alpha_{2}} & -\xi_{8} e^{-\beta_{1}} & \xi_{8} e^{\beta_{1}} \\
\psi_{3} & 0 & \psi_{4} & -\psi_{4} & \left(\frac{t_{n}}{s \delta}\right) & \left(\frac{t_{n}}{s \delta}\right) \\
\psi_{3} e^{-\alpha_{1}} & 0 & \psi_{4} e^{-\alpha_{2}} & -\psi_{4} e^{\alpha_{2}} & \left(\frac{t_{n}}{s \delta}\right) e^{-\beta_{1}} & \left(\frac{t_{n}}{s \delta}\right) e^{\beta_{1}}
\end{array}\right|,
$$

$\Delta_{3}=\left|\begin{array}{cccccc}\xi_{1} & \xi_{2} & \xi_{5} & \xi_{4} & 0 & 0 \\ \xi_{1} e^{-\alpha_{1}} & \xi_{2} e^{\alpha_{1}} & \xi_{5} & \xi_{4} e^{\alpha_{2}} & 0 & 0 \\ \psi_{1} & \psi_{1} & \xi_{5} \xi_{7} & \psi_{2} & -\xi_{8} & \xi_{8} \\ \psi_{1} e^{-\alpha_{1}} & \psi_{1} e^{\alpha_{1}} & \xi_{5} \xi_{7} & \psi_{2} e^{\alpha_{2}} & -\xi_{8} e^{-\beta_{1}} & \xi_{8} e^{\beta_{1}} \\ \psi_{3} & -\psi_{3} & 0 & -\psi_{4} & \left(\frac{t_{n}}{s \delta}\right) & \left(\frac{t_{n}}{s \delta}\right) \\ \psi_{3} e^{-\alpha_{1}} & -\psi_{3} e^{\alpha_{1}} & 0 & -\psi_{4} e^{\alpha_{2}} & \left(\frac{t_{n}}{s \delta}\right) e^{-\beta_{1}} & \left(\frac{t_{n}}{s \delta}\right) e^{\beta_{1}}\end{array}\right|$,

$$
\Delta_{4}=\left|\begin{array}{cccccc}
\xi_{1} & \xi_{2} & \xi_{3} & \xi_{5} & 0 & 0 \\
\xi_{1} e^{-\alpha_{1}} & \xi_{2} e^{\alpha_{1}} & \xi_{3} e^{-\alpha_{2}} & \xi_{5} & 0 & 0 \\
\psi_{1} & \psi_{1} & \psi_{2} & \xi_{5} \xi_{7} & -\xi_{8} & \xi_{8} \\
\psi_{1} e^{-\alpha_{1}} & \psi_{1} e^{\alpha_{1}} & \psi_{2} e^{-\alpha_{2}} & \xi_{5} \xi_{7} & -\xi_{8} e^{-\beta_{1}} & \xi_{8} e^{\beta_{1}} \\
\psi_{3} & -\psi_{3} & \psi_{4} & 0 & \left(\frac{t_{n}}{s \delta}\right) & \left(\frac{t_{n}}{s \delta}\right) \\
\psi_{3} e^{-\alpha_{1}} & -\psi_{3} e^{\alpha_{1}} & \psi_{4} e^{-\alpha_{2}} & 0 & \left(\frac{t_{n}}{s \delta}\right) e^{-\beta_{1}} & \left(\frac{t_{n}}{s \delta}\right) e^{\beta_{1}}
\end{array}\right|,
$$$$
\Delta_{5}=\left|\begin{array}{cccccc}
\xi_{1} & \xi_{2} & \xi_{3} & \xi_{4} & \xi_{5} & 0 \\
\xi_{1} e^{-\alpha_{1}} & \xi_{2} e^{\alpha_{1}} & \xi_{3} e^{-\alpha_{2}} & \xi_{4} e^{\alpha_{2}} & \xi_{5} & 0 \\
\psi_{1} & \psi_{1} & \psi_{2} & \psi_{2} & \xi_{5} \xi_{7} & \xi_{8} \\
\psi_{1} e^{-\alpha_{1}} & \psi_{1} e^{\alpha_{1}} & \psi_{2} e^{-\alpha_{2}} & \psi_{2} e^{\alpha_{2}} & \xi_{5} \xi_{7} & \xi_{8} e^{\beta_{1}} \\
\psi_{3} & -\psi_{3} & \psi_{4} & -\psi_{4} & 0 & \left(\frac{t_{n}}{s \delta}\right) \\
\psi_{3} e^{-\alpha_{1}} & -\psi_{3} e^{\alpha_{1}} & \psi_{4} e^{-\alpha_{2}} & -\psi_{4} e^{\alpha_{2}} & 0 & \left(\frac{t_{n}}{s \delta}\right) e^{\beta_{1}}
\end{array}\right|,
$$

$$
\Delta_{6}=\left|\begin{array}{cccccc}
\xi_{1} & \xi_{2} & \xi_{3} & \xi_{4} & 0 & \xi_{5} \\
\xi_{1} e^{-\alpha_{1}} & \xi_{2} e^{\alpha_{1}} & \xi_{3} e^{-\alpha_{2}} & \xi_{4} e^{\alpha_{2}} & 0 & \xi_{5} \\
\psi_{1} & \psi_{1} & \psi_{2} & \psi_{2} & -\xi_{8} & \xi_{5} \xi_{7} \\
\psi_{1} e^{-\alpha_{1}} & \psi_{1} e^{\alpha_{1}} & \psi_{2} e^{-\alpha_{2}} & \psi_{2} e^{\alpha_{2}} & -\xi_{8} e^{-\beta_{1}} & \xi_{5} \xi_{7} \\
\psi_{3} & -\psi_{3} & \psi_{4} & -\psi_{4} & \left(\frac{t_{n}}{s \delta}\right) & 0 \\
\psi_{3} e^{-\alpha_{1}} & -\psi_{3} e^{\alpha_{1}} & \psi_{4} e^{-\alpha_{2}} & -\psi_{4} e^{\alpha_{2}} & \left(\frac{t_{n}}{s \delta}\right) e^{-\beta_{1}} & 0
\end{array}\right|
$$

Where

$$
\begin{gathered}
\psi_{1}=\xi_{6} \alpha_{1}^{2}+\xi_{7}, \quad \psi_{2}=\xi_{6} \alpha_{2}^{2}+\xi_{7}, \quad \psi_{3}=\alpha_{1}+\frac{\alpha_{1}^{3}}{s c_{0}}-\xi_{9} \alpha_{1}, \\
\psi_{3}=\alpha_{2}+\frac{\alpha_{2}^{3}}{s c_{0}}-\xi_{9} \alpha_{2}, \\
\xi_{1}=1+\alpha_{1} \beta_{v} k_{m}, \quad \xi_{2}=1-\alpha_{1} \beta_{v} k_{m}, \quad \xi_{3}=1+\alpha_{2} \beta_{v} k_{m}, \\
\xi_{4}=1-\alpha_{2} \beta_{v} k_{m}, \\
\xi_{5}=\frac{2(-1)^{n} L}{h a_{2} t_{n}}, \quad \xi_{6}=\frac{-t_{n}}{s c_{0}}, \xi_{7}=-t_{n}+\frac{t_{n}^{3}}{s c_{0}}+\frac{t_{n} M^{2}}{s c_{0}}+\frac{t_{n}}{s c_{0} k_{p}^{*}}, \quad \xi_{8}=\frac{\beta_{1}}{s \delta}, \\
\xi_{9}=\frac{t_{n}^{2}}{s c_{0}}+\frac{M^{2}}{s c_{0}}+\frac{1}{s c_{0} k_{p}^{*}} .
\end{gathered}
$$

\section{Results and Discussion}

The slip flow of micropolar fluid through a porous medium in a rectangular microchannel affected by a uniform magnetic field is investigated. The differential equations which governing this 
phenomenon and subjected to the appropriate boundary conditions have been solved analytically by using Fourier series. The effect of a physical parameter is discussed numerically and illustrated graphically through a set of Figures 2-22.

The variation of velocity $c$ for different values of the parameters is shown in Figures 2-7. The contour lines of $u$ are shown in Figures $2 \mathrm{~b}$ and $3 \mathrm{~b}$, and flow pattern of $u$ are shown in Figures $2 \mathrm{a}$ and $3 \mathrm{a}$ for different values of magnetic parameter $M$. Figure $2 c$ illustrate the variation of the velocity $u$ with the magnetic parameter $M$, we observe
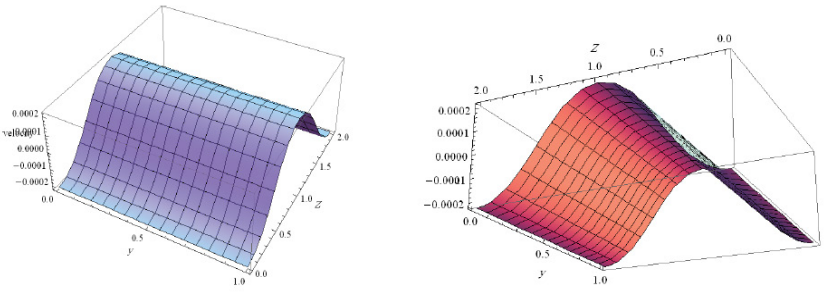

Figure 2a: Velocity profile of $u$ in $3 D$ at $M=10$.

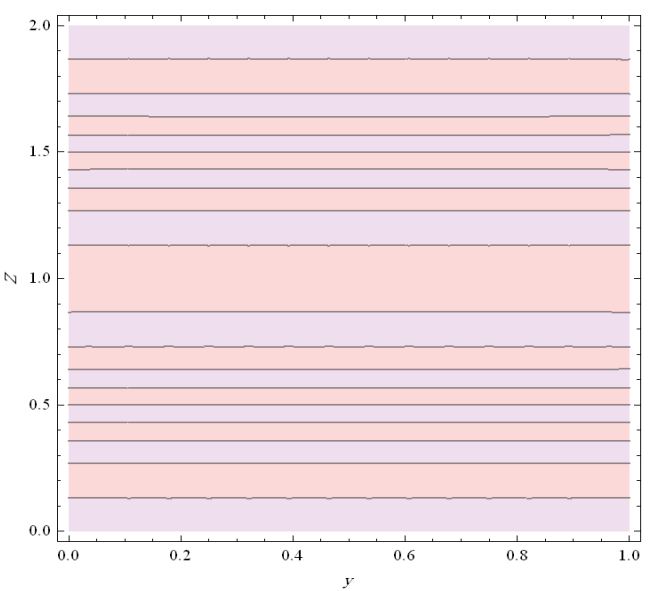

Figure 2b: Contours of $u$ at $M=10$.

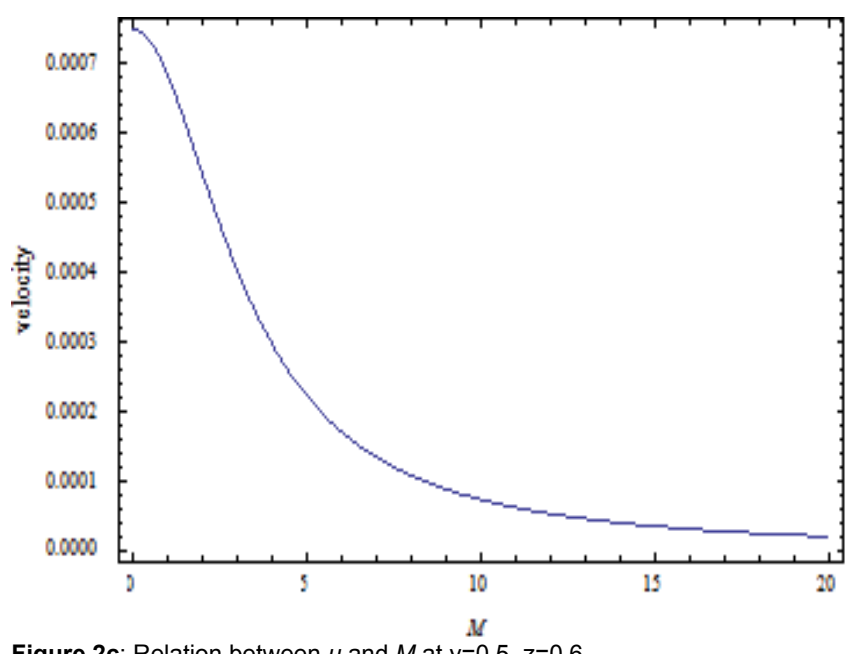

Figure 2c: Relation between $u$ and $M$ at $\mathrm{y}=0.5, \mathrm{z}=0.6$

At: $L=0.2, s=0.3, h=0.5, k_{p}^{*}=3, c_{0}=0.1, \delta=0.4, \beta_{v}=0.1, k_{m}=0.25$
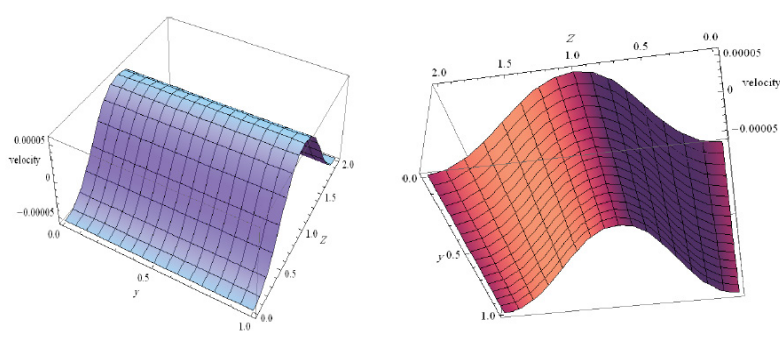

Figure 3a: Velocity profile of $u$ in $3 D$ at $M=20$.

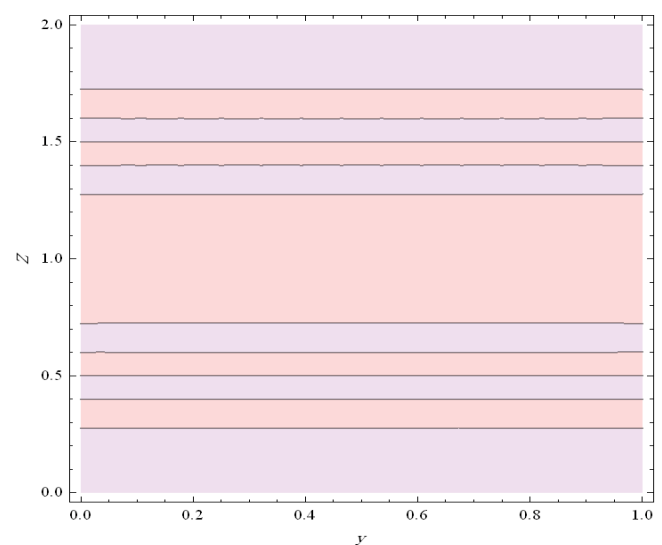

Figure 3b: Contours of $u$ at $M=20$.

At: $L=0.2, s=0.3, h=0.5, k_{p}^{*}=3, c_{0}=0.1, \delta=0.4, \beta_{v}=0.1, k_{m}=0.25$

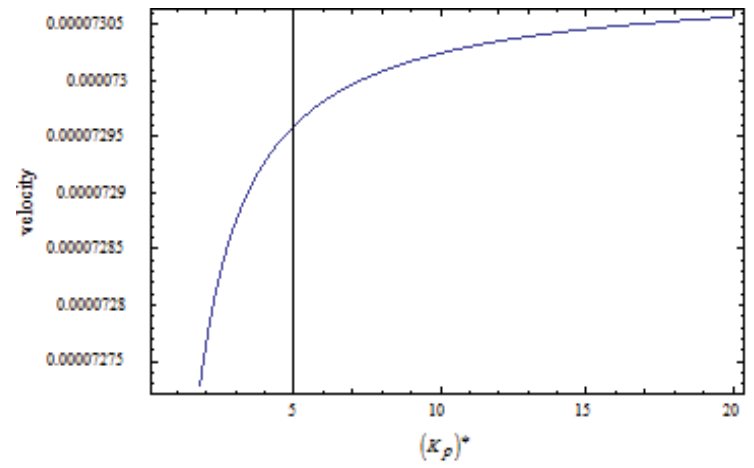

Figure 4a: Relation between $u$ and $k_{p}^{*}$ at $\mathrm{y}=0.5, \mathrm{z}=0.6, \mathrm{~s}=0.3$.

At: $L=0.2, h=0.5, c_{0}=0.1, \delta=0.4, \beta_{v}=0.1, k_{m}=0.25$

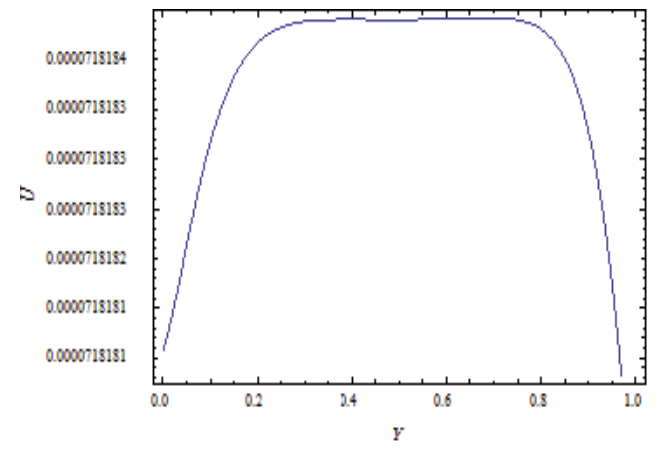

Figure 4b: Velocity profile of $u$ and $k_{p}^{*}=0.5$ 


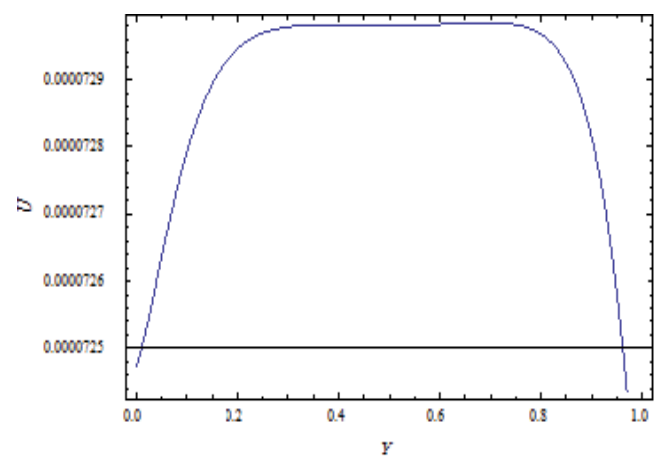

Figure 4c: Velocity profile of $u$ and $k_{p}^{*}=6$

At: $L=0.2, s=0.3, h=0.5, M=10, c_{0}=0.1, \delta=0.4, \beta_{v}=0.1, k_{m}=0.25, z=0.6$

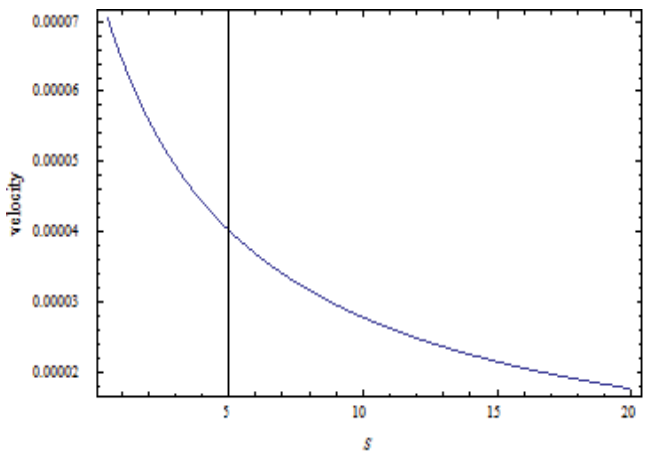

Figure 5a: Relation between and $s y=0.5, z=0.6, k_{p}^{*}=3$.

At: $L=0.2, s=0.3, h=0.5, k_{p}^{*}=3, c_{0}=0.1, \delta=0.4, \beta_{v}=0.1, k_{m}=0.25$

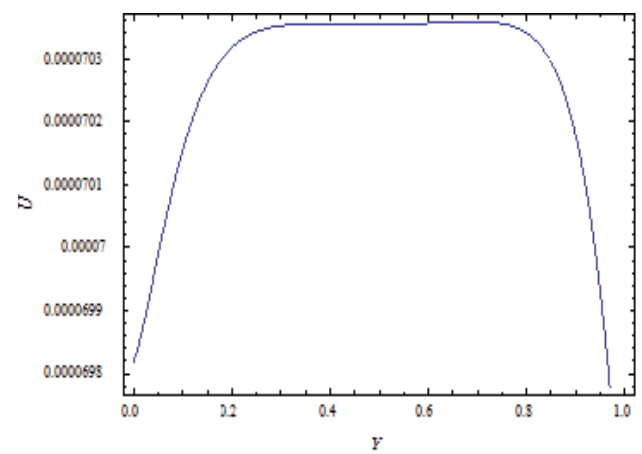

Figure 5b: Velocity profile of $u$ at $s=0.5$

that the velocity $u$ decreases with increase in the magnetic parameter $M$. It is observed from Figure $4 \mathrm{a}$ that the velocity $u$ increases for increasing the porous parameter $k_{p}^{*}$. Figures $4 \mathrm{~b}$ and $4 \mathrm{c}$ illustrates the distribution of the velocity $u$, with $y$ direction for different values of $k_{p}^{*}$, it can have observed that the velocity $u$ is increasing. Figure 5 a illustrates the velocity profile decrease for increasing the couple stress parameter $s$. The variation of velocity $u$, with $y$ direction for different values of $s$ is shown in Figures $5 \mathrm{~b}$ and $5 \mathrm{c}$. From Figure $6 \mathrm{a}$, it can be observed that the velocity $u$ is increasing as the coupling parameter $c_{0}$ increasing. Figures $6 \mathrm{~b}$ and $6 \mathrm{c}$ indicate that with an increase in the value of $s$, the velocity $u$ increase with $y$ direction. The velocity $u$ at various Kundsen number $k_{m}$ are shown in Figures $7 \mathrm{a}, 7 \mathrm{~b}$ and $7 \mathrm{c}$, we observe that the velocity $u$ increases when Kundsen number $k_{m}$ increases.

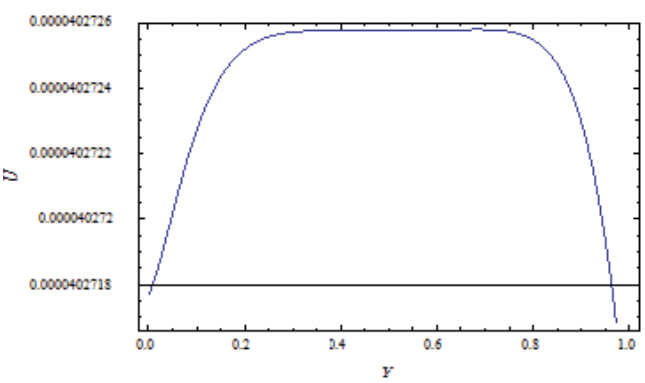

Figure 5c: Velocity profile of $u$ at $\mathrm{s}=0.5$

At: $L=0.2, k_{p}^{*}=3, h=0.5, M=10, c_{0}=0.1, \delta=0.4, \beta_{v}=0.1, k_{m}=0.25, z=0.6$

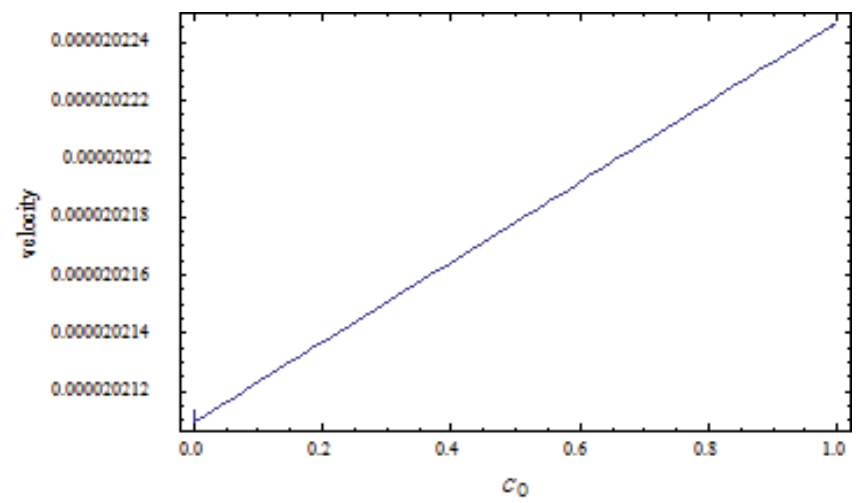

Figure 6a: Relation between $u$ and $c_{0}$ at $\mathrm{y}=0.5, \mathrm{z}=0.6, \mathrm{k}_{\mathrm{m}}=0.25$

At: $L=0.2, h=0.5, M=20, \delta=0.4, \beta_{v}=0.1, k_{p}^{*}=3, s=0.3$

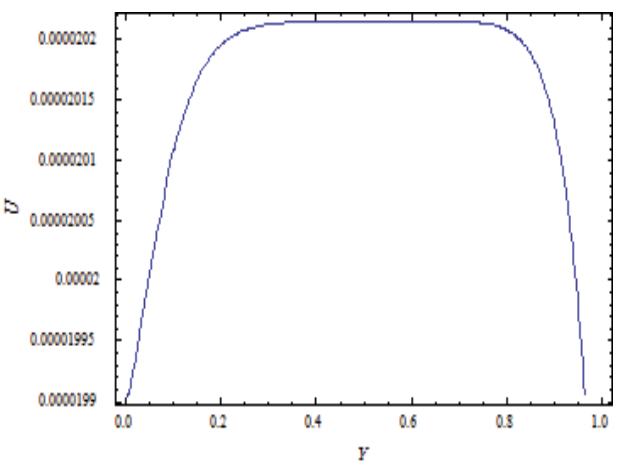

Figure $6 \mathbf{b}$ : Velocity profile of $u$ at $c_{0=} 0.3$

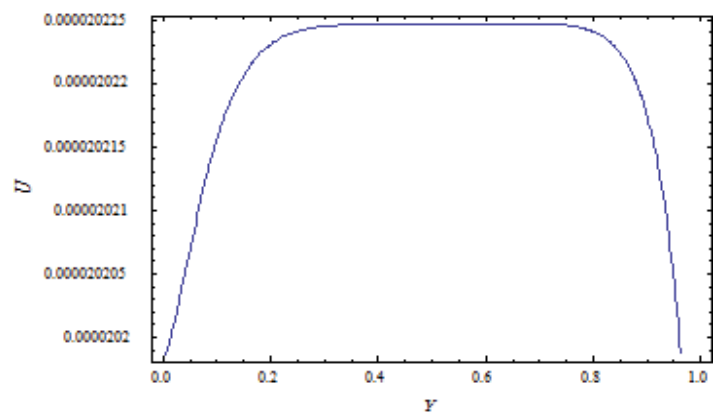

Figure 6c: Velocity profile of $u$ at $c_{0}=1$

At: $L=0.2, k_{p}^{*}=3, h=0.5, M=20, s=0.3, \delta=0.4, \beta_{v}=0.1, k_{m}=0.25, z=0.6$ 


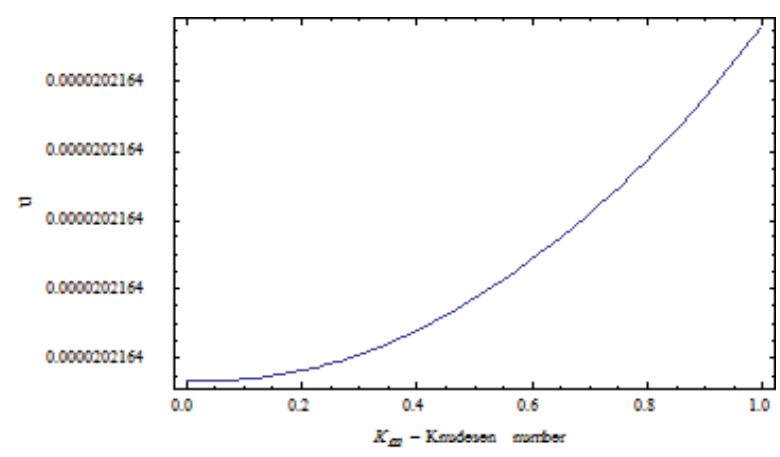

Figure 7a: Relation between $u$ and $k_{m}$ at $y=0.5, z=0.6, c_{0}=0.4$ At: $L=0.2, h=0.5, M=20, \delta=0.4, \beta_{v}=0.1, k_{p}^{*}=3, s=0.3$

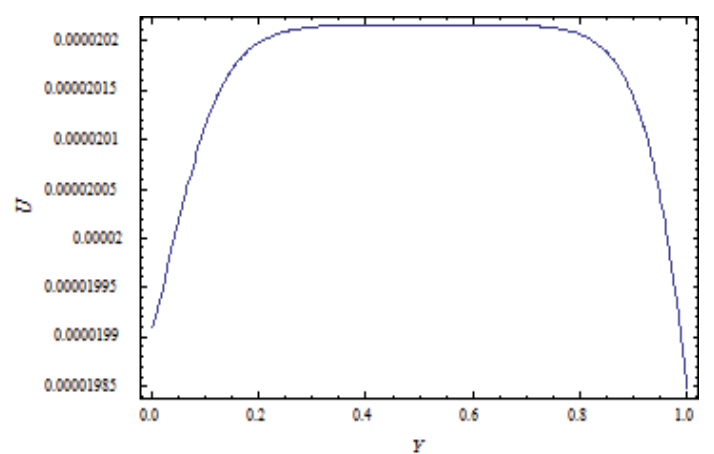

Figure $7 \mathbf{b}$ : Velocity profile of $u$ at $k_{\mathrm{m}}=0.1$.

At: $L=0.2, h=0.5, M=20, \delta=0.4, \beta_{v}=0.1, k_{p}^{*}=3, s=0.3$

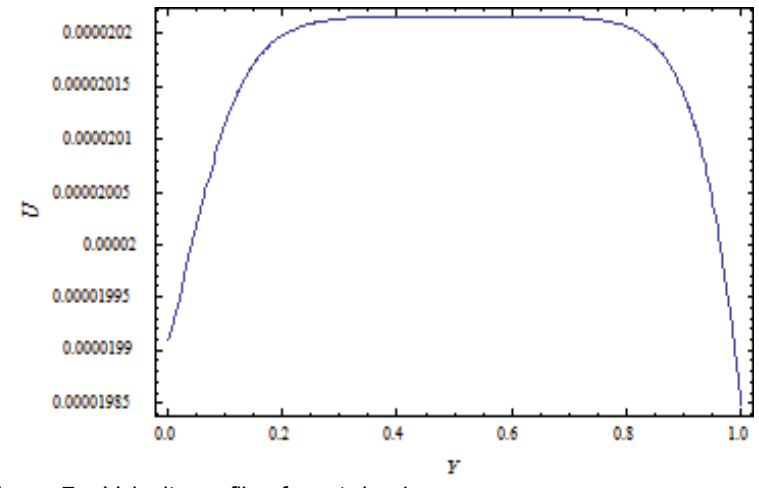

Figure 7c: Velocity profile of $u$ at $k_{\mathrm{m}}=1$.

At: $L=0.2, k_{p}^{*}=3, h=0.5, M=20, s=0.3, \delta=0.4, \beta_{v}=0.1, c_{0}=0.3, z=0.6$

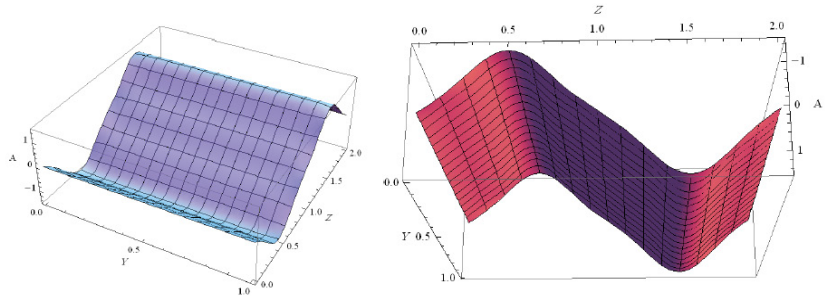

Figure 8a: Microrotation component $A$ (of $y$ - direction) in $3 D$ at $M=10$

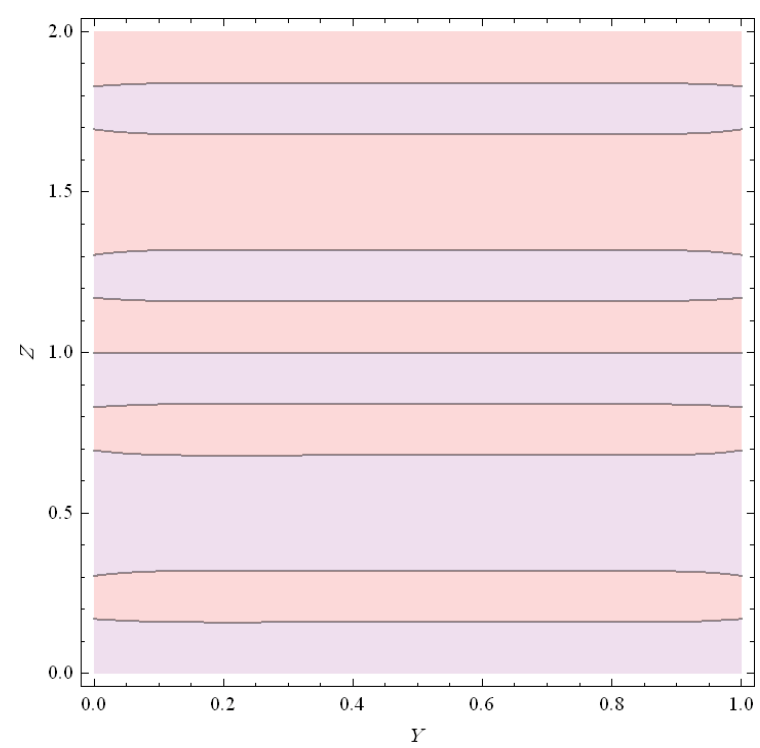

Figure 8b: Contours of $\mathrm{A}$ at $M=10$.

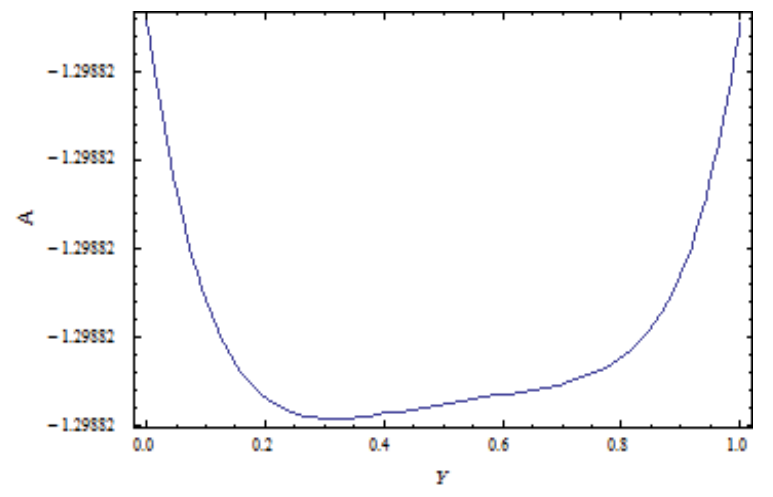

Figure 8c: Microrotation $\mathrm{A}$ at $M=10$

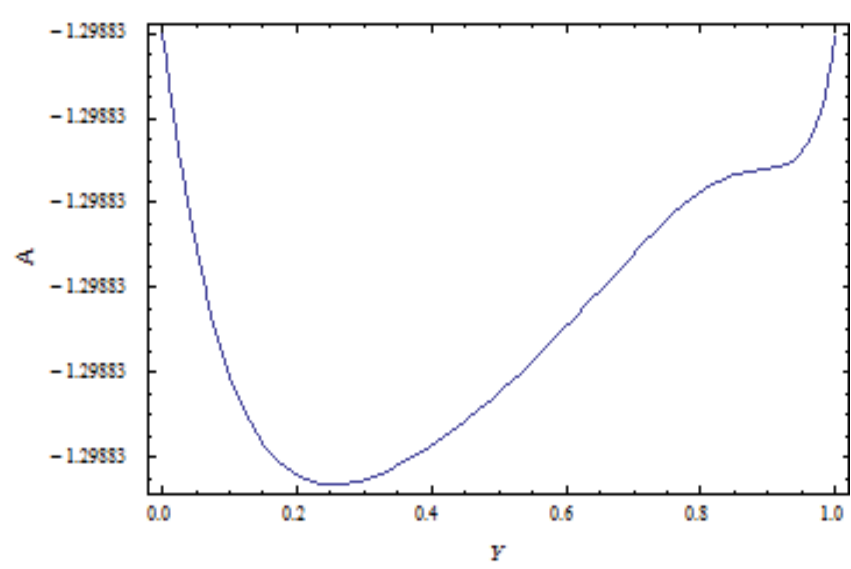

Figure 8d: Microrotation $A$ at $M=20$

Figures 8-12 illustrate the variation of the microrotation $y$ component $c$ for different values of $M, k_{p}^{*}, c_{0}, s$ and $k_{m}$. Figure 8a 


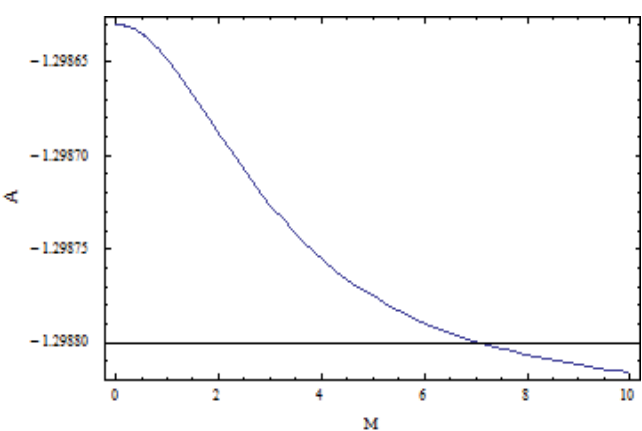

Figure 8e: Relation between $A$ and $M$ at $y=0.6$

At: $L=0.2, k_{p}^{*}=3, h=0.5, k_{m}=0.25, s=0.3, \delta=0.4, \beta_{v}=0.1, c_{0}=0.1, z=0.6$

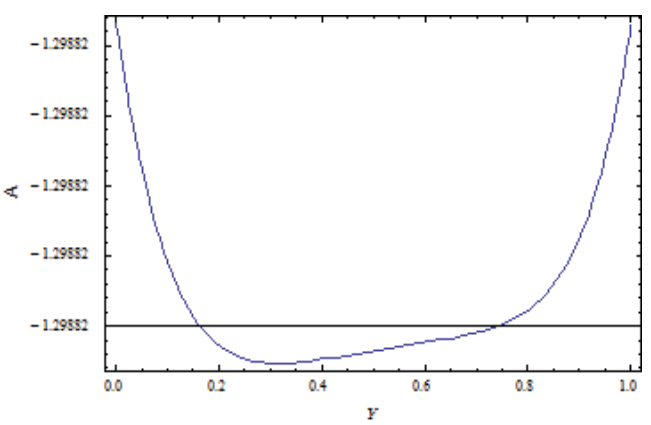

Figure 9a: Microrotation A at $k_{p}^{*}=0.1$.

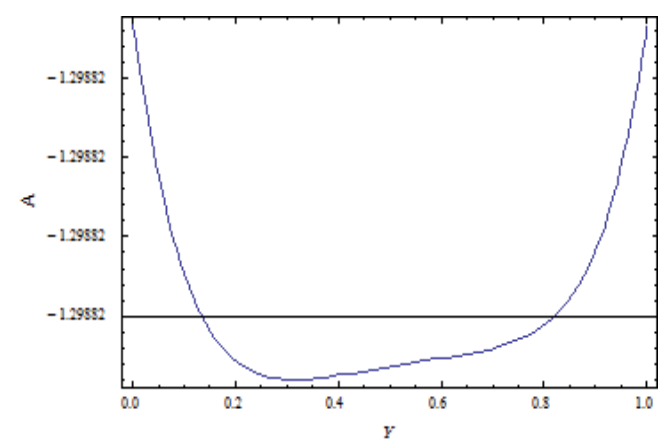

Figure 9b: Microrotation A at $k_{p}^{*}=100$

At: $L=0.2, M=10, h=0.5, k_{m}=0.25, s=0.3, \delta=0.4, \beta_{v}=0.1, c_{0}=0.1, z=0.6$

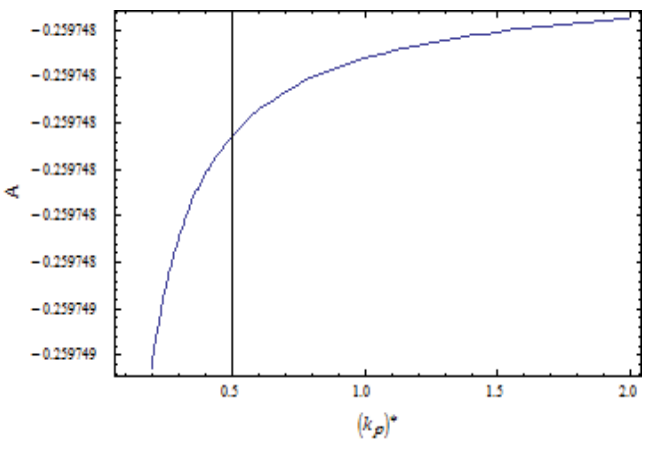

Figure 9c: Relation between $\mathrm{A}$ and $k_{p}^{*}$ at $y=0.1$.

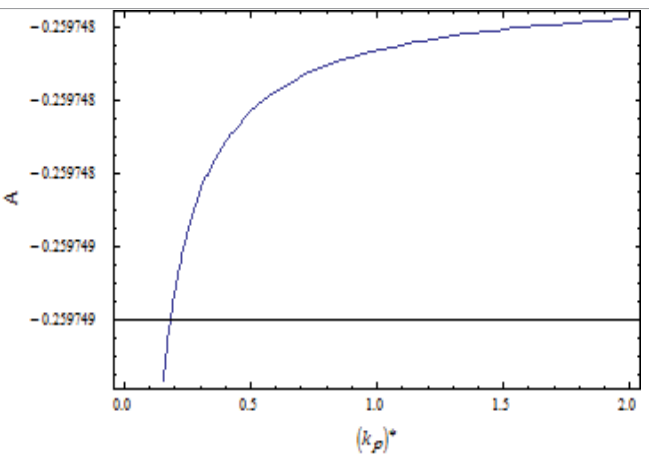

Figure 9d: Relation between $A$ and $k_{p}^{*}$ at $y=0.8$

At: $L=0.2, M=10, h=0.5, s=0.3, c_{0}=0.5, \delta=0.4, \beta_{v}=10, k_{m}=0.8, z=0.6$

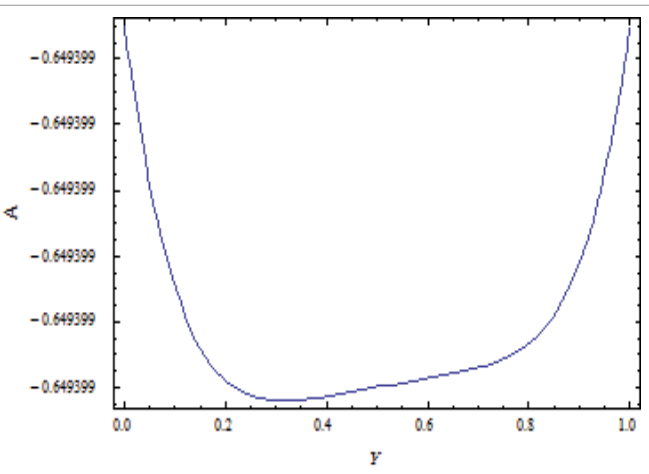

Figure 10a: Microrotation $A$ at $\mathrm{c}_{0=} 0.2$.

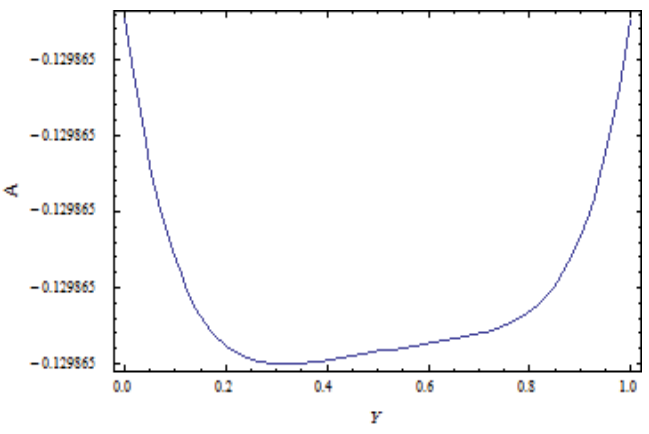

Figure 10b: Microrotation $A$ at $\mathrm{c}_{0=} 1$.

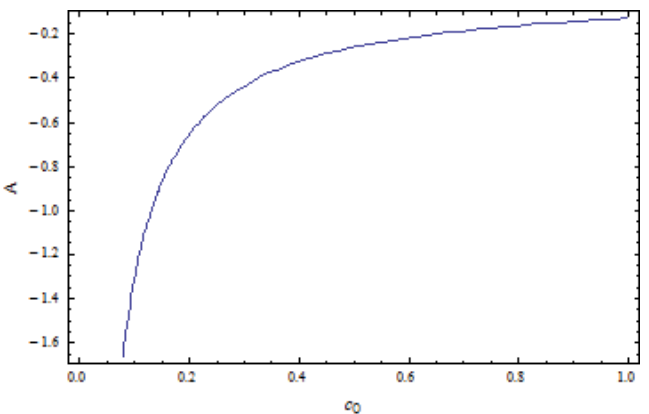

Figure 10c: Relation between $\mathrm{A}$ and $c_{0}$ at $y=0.6$.

At: $L=0.2, M=10, h=0.5, k_{m}=0.25, s=0.3, \delta=0.4, \beta_{v}=0.1, k_{p}^{*}=0.5, z=0.6$ 
Citation: Agoor BM (2018) Slip Flow in Porous Medium of Micropolar Fluid in a Rectangular Microchannel under the Effect of a Magnetic Field. J Appl Mech Eng 7: 298. doi:10.4172/2168-9873.1000298

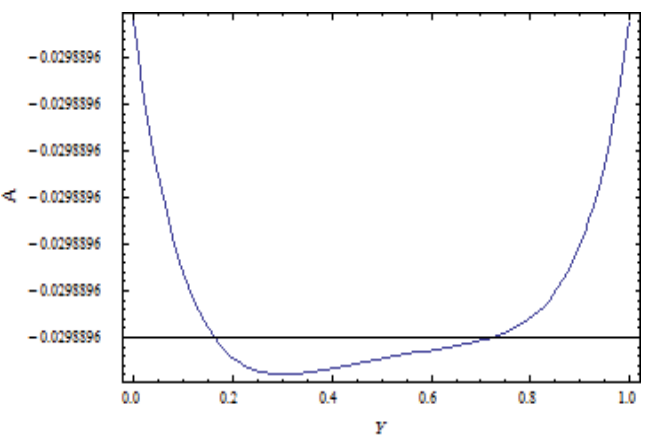

Figure 11a: Microrotation $A$ at $S=2$

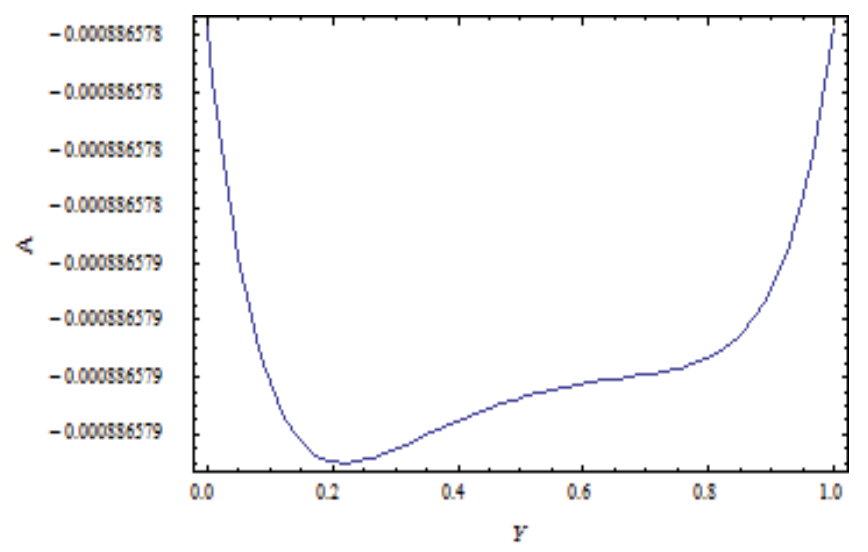

Figure 11b: Microrotation $A$ at $S=20$.

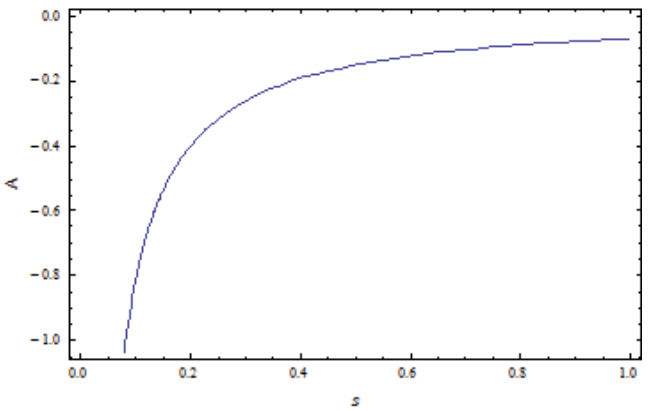

Figure 11c: Relation between $A$ and $S$ at $\mathrm{y}=0.6$.

At: $L=0.2, M=10, h=0.5, k_{m}=0.25, c_{0}=0.5, \delta=0.4, \beta_{v}=0.1, k_{p}^{*}=0.5, z=0.6$

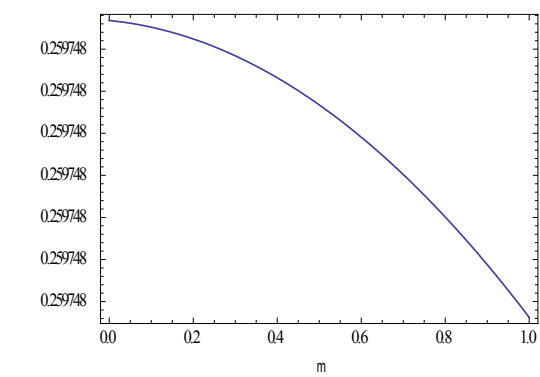

Figure 12a: Relation between $\mathrm{A}$ and $k_{m}$ at $\mathrm{y}=0.3$.

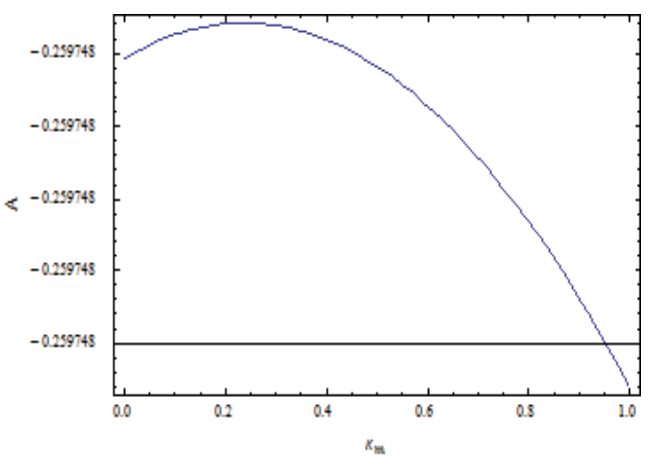

Figure 12b: Relation between $\mathrm{A}$ and $k$ at $\mathrm{y}=0.7$

At: $L=0.2, M=10, h=0.5, s=0.3, c_{0}=0.5, \delta=0.4, \beta_{v}=0.1, k_{p}^{*}=0.5, z=0.6$

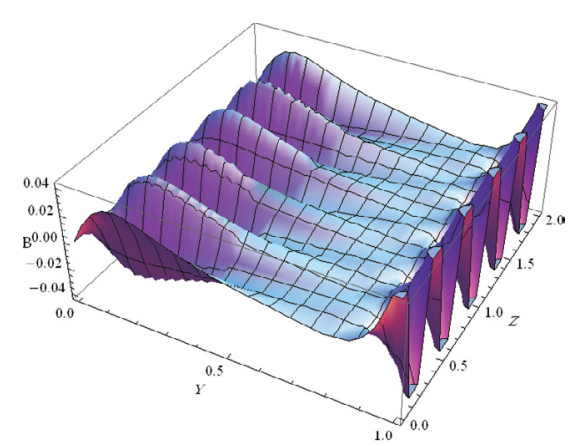

Figure 13a: Micro-rotation component $B$ (of $Z$ - direction) in $3 D$ at $M=0$.

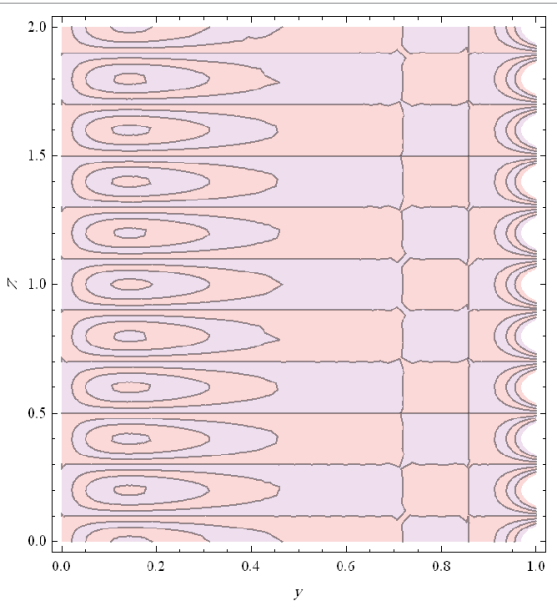

Figure 13b: Contours of $B$ at $M=0$.

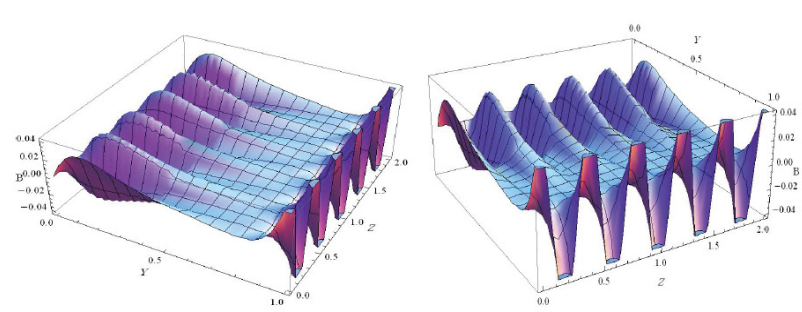

Figure 13c: Microrotation component $B$ (of $z$ - direction) in 3D at $M=10$. 


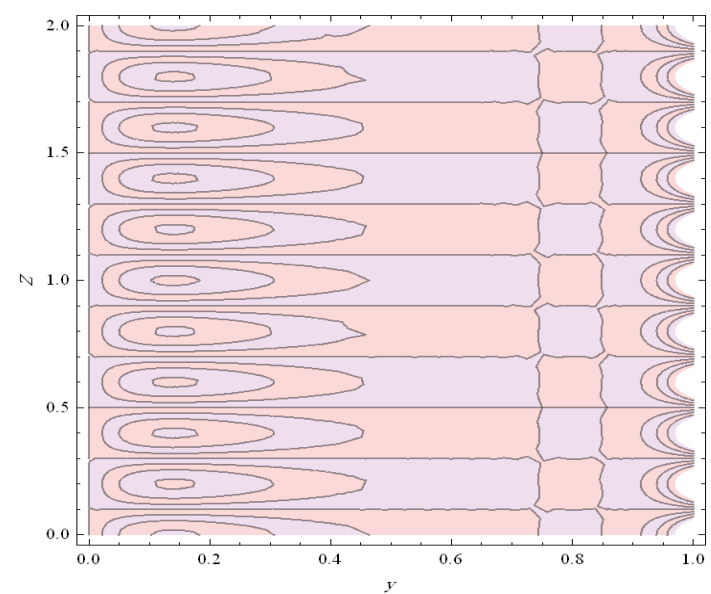

Figure 13d: Contours of $\mathrm{B}$ at $M=10$.

At: $L=0.2, k_{p}^{*}=3, h=0.5, k_{m}=0.25, s=0.3, \delta=0.4, \beta_{v}=0.1, c_{0}=0.1$

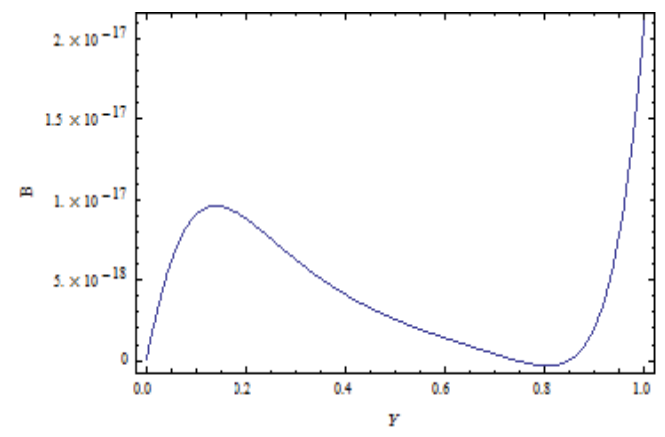

Figure 14a: Microrotation $B$ at $M=10$.

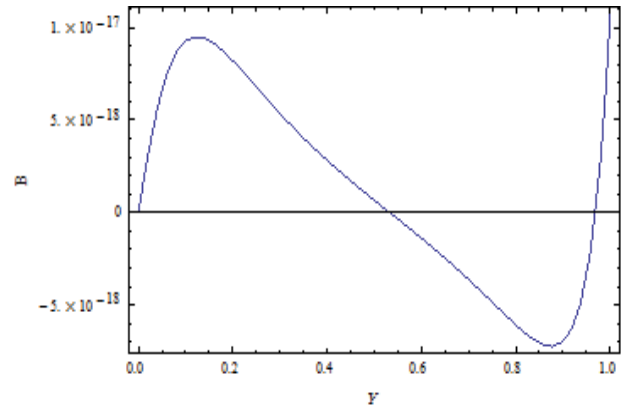

Figure 14b: Microrotation $B$ at $M=30$.

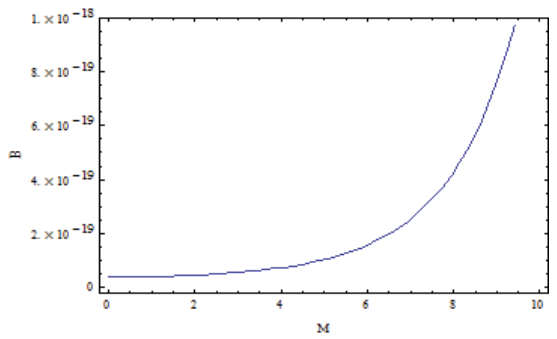

Figure 14c: Relation between $B$ and $M$ at $y=0.6$.

At: $L=0.2, k_{p}^{*}=3, h=0.5, k_{m}=0.25, s=0.3, \delta=0.4, \beta_{v}=0.1, c_{0}=0.1, z=0.5$

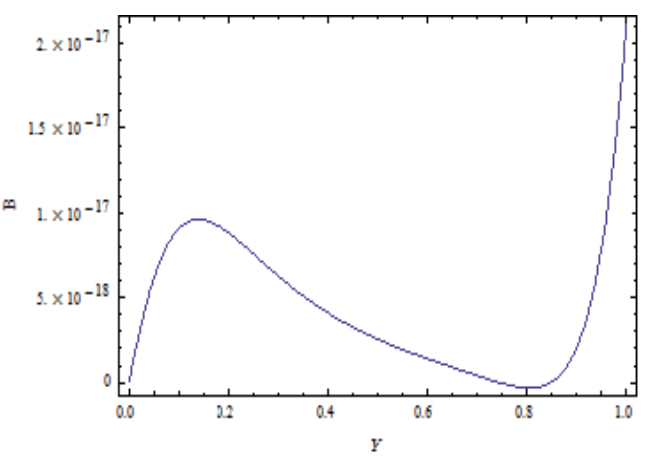

Figure 15a: Microrotation $B$ at $k_{p}^{*}=8$.

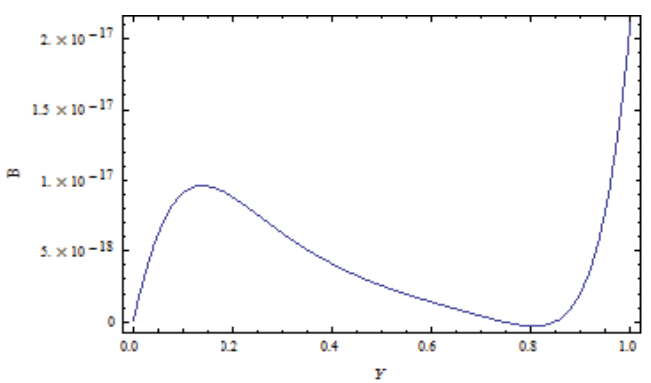

Figure 15b: Microrotation $B$ at $k_{p}^{*}=0.2$

At: $L=0.2, M=10, h=0.5, k_{m}=0.25, s=0.3, \delta=0.4, \beta_{v}=0.1, c_{0}=0.1, z=0.5$

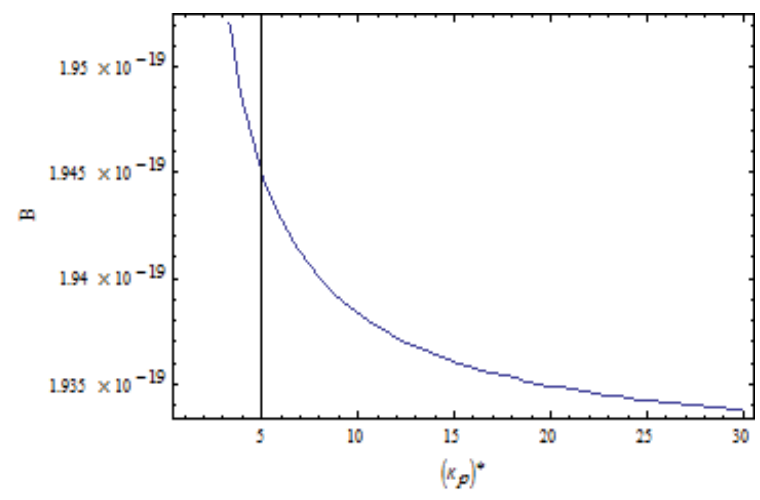

Figure 15c: Relation between $B$ and $k_{p}^{*}$ at $y=0.8$.

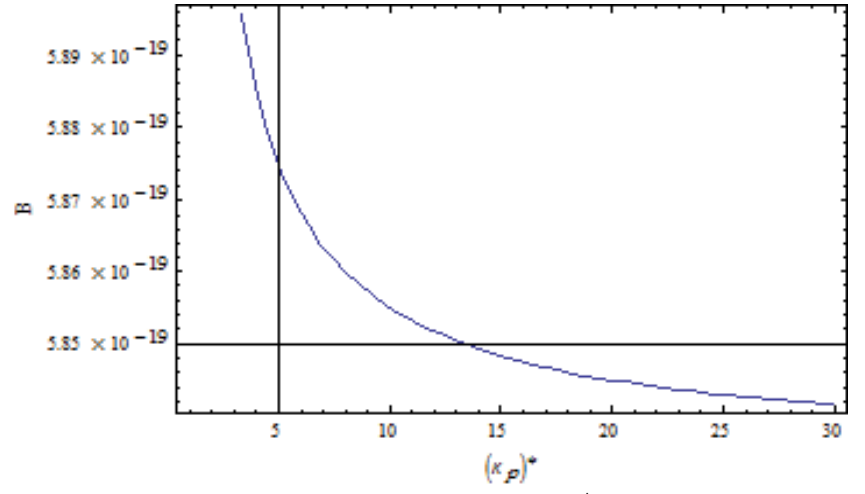

Figure 15d: Relation between $B$ and $k_{p}^{*}$ at $y=0.3$. 


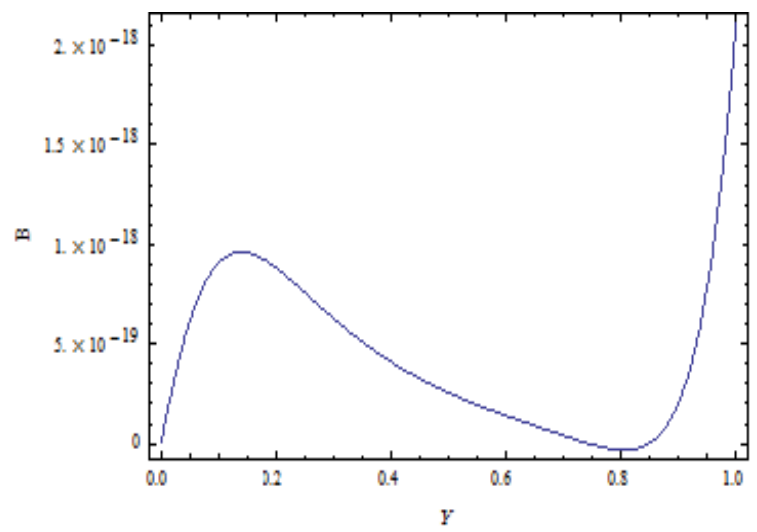

Figure 16a: Microrotation $B$ at $c_{0}=1$.

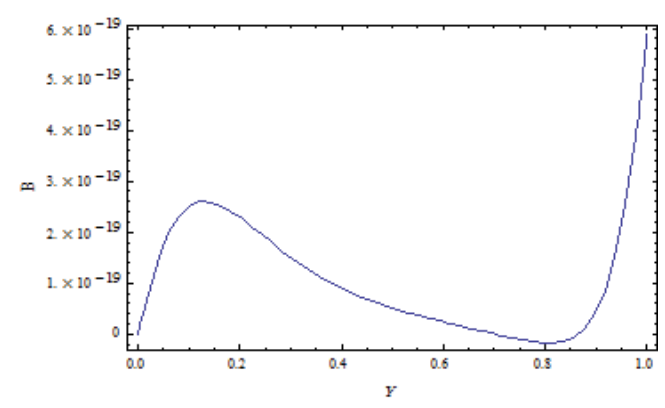

Figure 16b: Microrotation $B$ at $\mathrm{c}_{0}=0.1$.

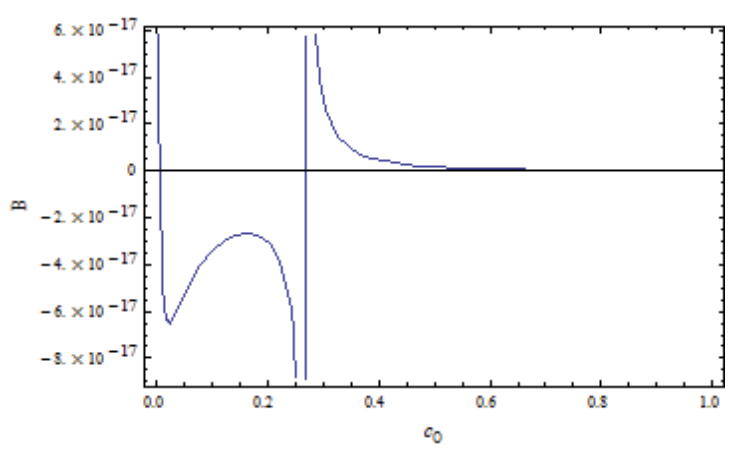

Figure 16c: Relation between $B$ and $c_{0}$ at $y=0.6$

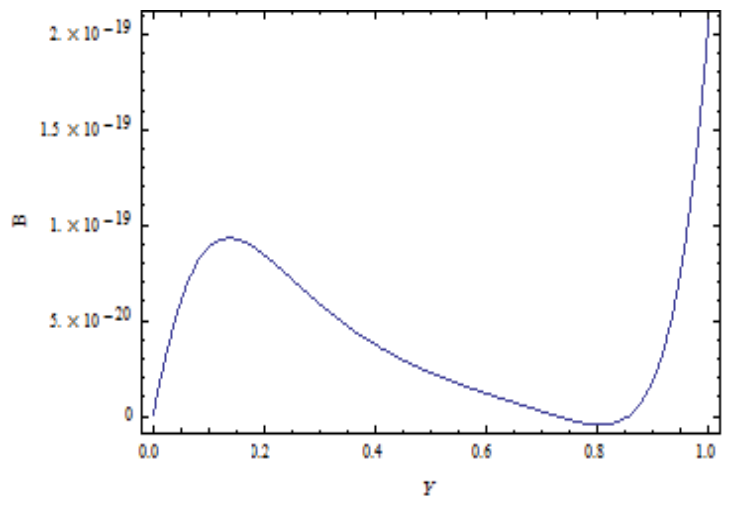

Figure 17a: Microrotation $B$ at $S=3$

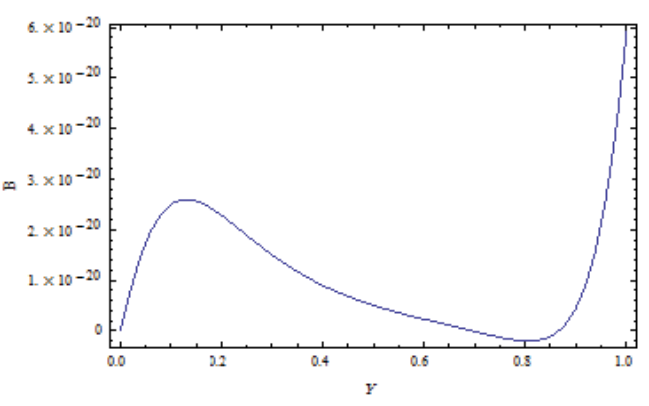

Figure 17b: Microrotation $B$ at $S=10$.

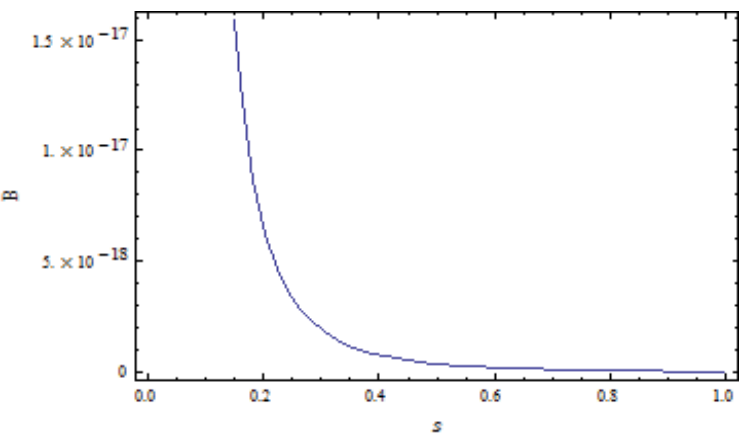

Figure 17c: Relation between $B$ and $s$ at $\mathrm{y}=0.6$.

At: $L=0.2, M=10, h=0.5, k_{m}=0.25, c_{0}=1, \delta=0.4, \beta_{v}=0.1, k_{p}^{*}=3, z=0.5$

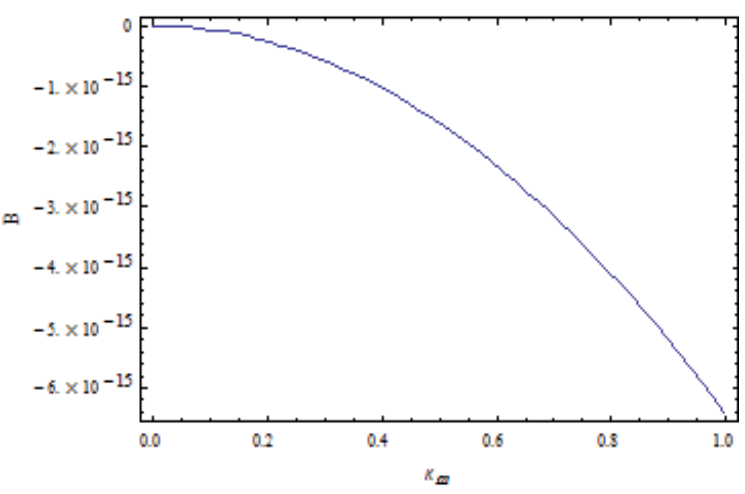

Figure 18a: Relation between $B$ and $k_{m}$ at $\mathrm{y}=0.8$.

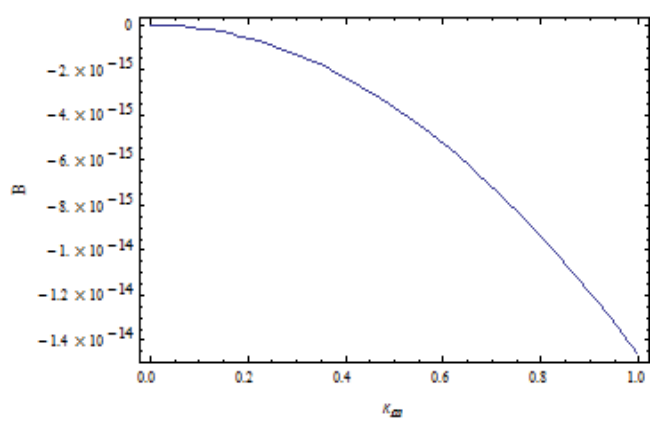

Figure 18b: Relation between $B$ and $k_{m}$ at $\mathrm{y}=0.3$.

At: $L=0.2, M=10, h=0.5, s=0.3, c_{0}=1, \delta=0.4, \beta_{v}=8, k_{p}^{*}=7, z=0.5$ 


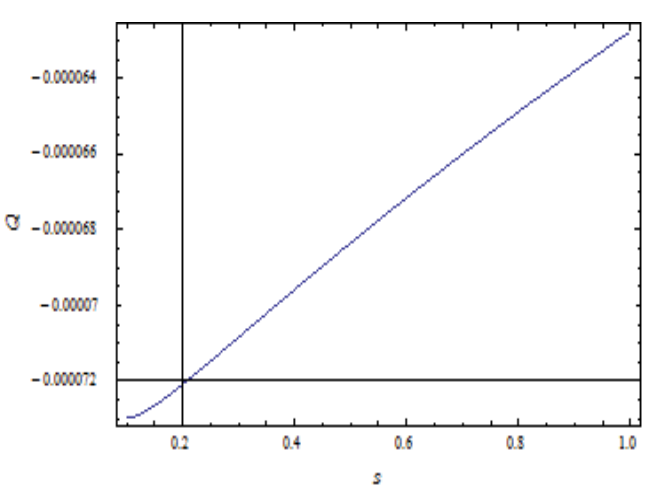

Figure 19a: Volumetric flow rate $Q$ with $S$, at $M=10$.

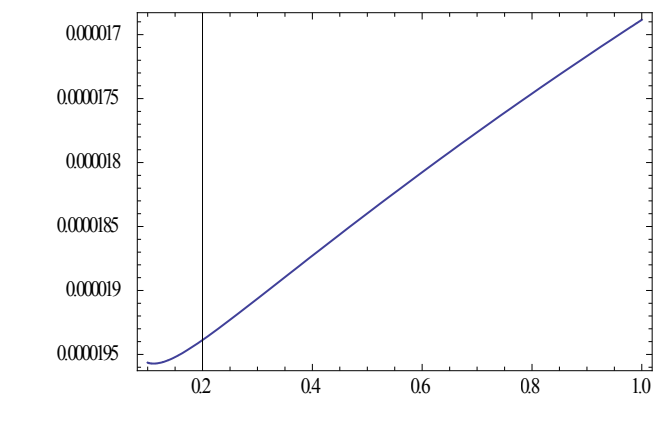

Figure 19b: Volumetric flow rate $Q$ with $S$, at $\mathrm{M}=20$.

At: $L=0.2, h=0.5, s=0.3, c_{0}=0.5, \delta=0.4, \beta_{v}=0.1, k_{m}=0.25, k_{p}^{*}=3$

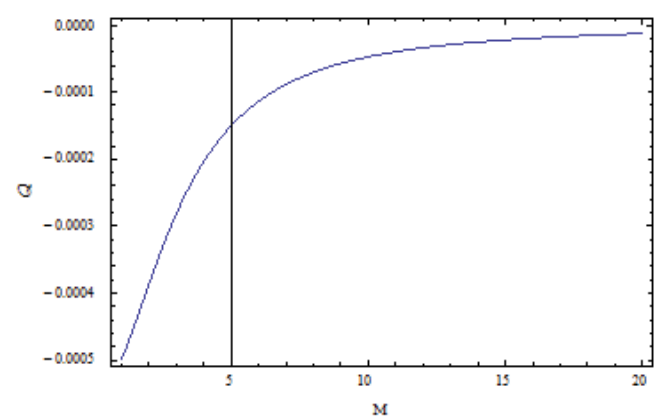

Figure 19c: Relation between volumetric flow rate $Q$ and $M$. At: $L=0.2, h=0.5, s=3, c_{0}=0.5, \delta=0.4, \beta_{v}=0.1, k_{m}=0.25, k_{p}^{*}=3$

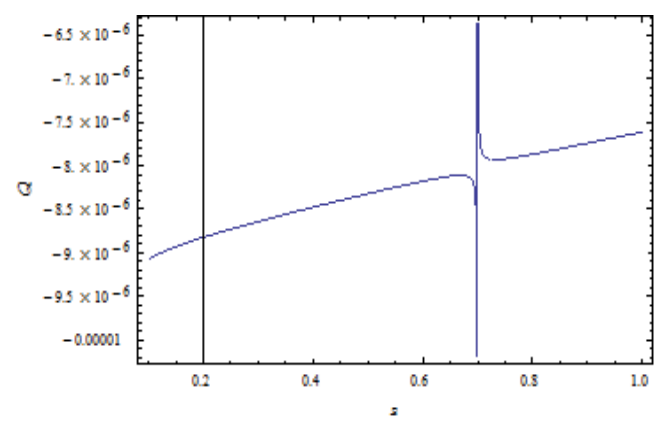

Figure 20a: Volumetric flow rate $Q$ with $S$, at $\mathrm{c}_{0=} 0.5$

At: $L=0.2, h=0.5, s=0.3, M=30, \delta=0.4, \beta_{v}=0.1, k_{m}=0.25, k_{p}^{*}=3$

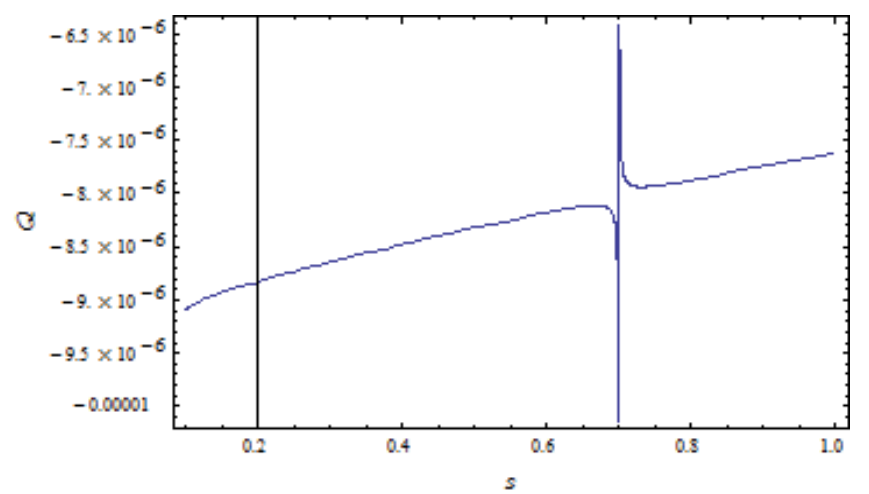

Figure 20b: Volumetric flow rate $Q$ with $S$, at $\mathrm{c}_{0}=1$

At: $L=0.2, h=0.8, s=3, M=10, \delta=0.4, \beta_{v}=0.1, k_{m}=0.25, k_{p}^{*}=3$

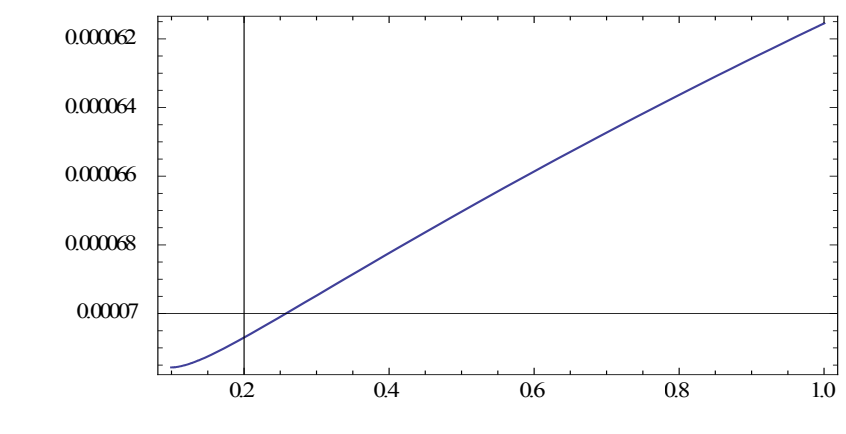

Figure 20c: Volumetric flow rate $Q$ with $S$, at $k_{p}^{*}=0.4$

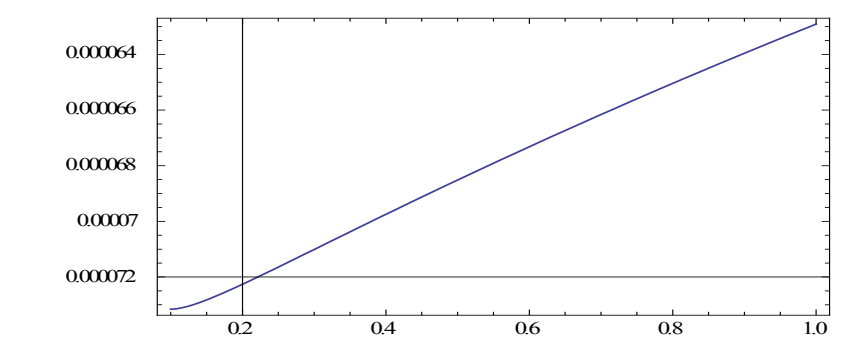

Figure 20d: Volumetric flow rate $Q$ with $\mathrm{S}$, at $k_{p}^{*}=15$

At: $L=0.2, h=0.5, c_{0}=0.5, M=10, \delta=0.4, \beta_{v}=0.1, k_{m}=0.25, k_{p}^{*}=0.4$
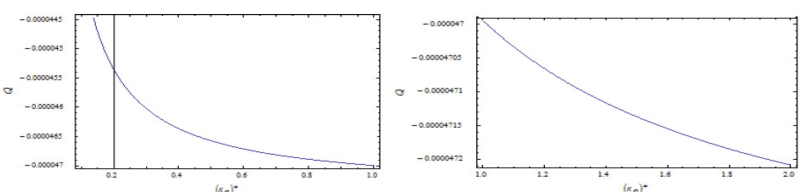

Figure 21a: Relation between volumetric flow rate $Q$ and $k^{*}$

At: $L=0.2, h=0.5, c_{0}=0.5, M=10, \delta=0.4, \beta_{v}=0.1, k_{m}=0.25, k_{p}^{*}=0.4, s=3$

illustrates the flow pattern of $A$ and the contour lines of $A$ is shown in Figure $8 \mathrm{~b}$. The variation of $A$, with $y$ direction for different values of $M$ is shown in Figures $8 \mathrm{c}$ and $8 \mathrm{~d}$. From Figure 8e, it can be observed that the microrotation $y$ component $A$ decreases with increase in the magnetic parameter $M$. Figures $9 \mathrm{a}$ and $9 \mathrm{~b}$ illustrate the variation of $A$ 


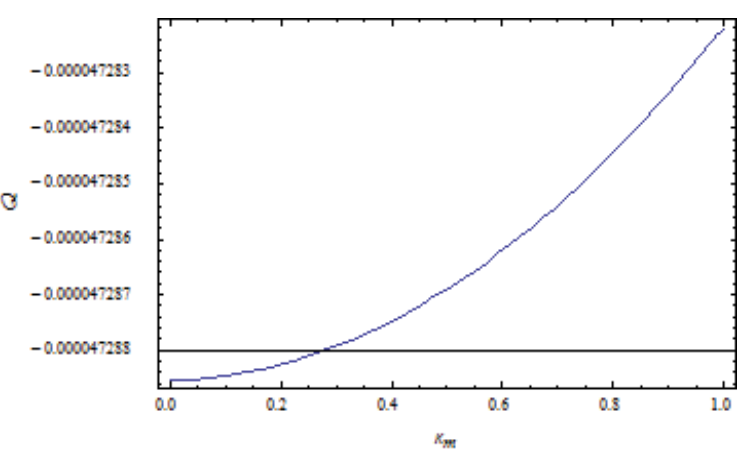

Figure 21b: Relation between Volumetric flow rate $Q$ and $k_{m}$. At: $L=0.2, h=0.5, s=3, M=10, \delta=0.4, \beta_{v}=0.1, k_{p}^{*}=3, c_{0}=0.5$

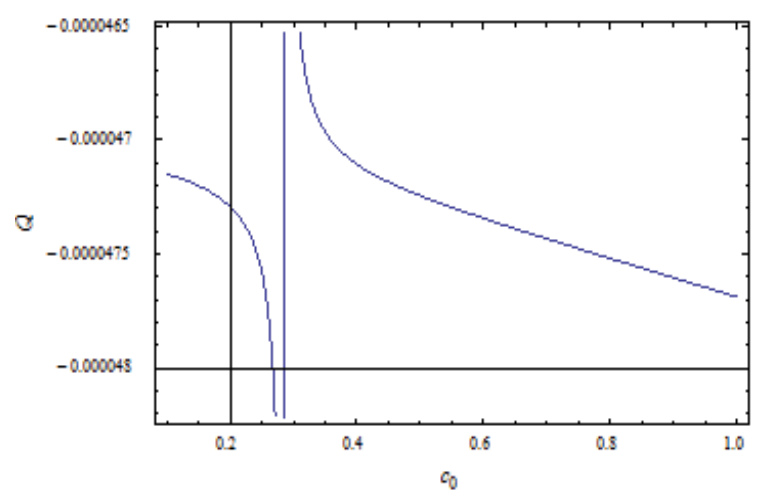

Figure 22a: Volumetric flow rate $Q$ with $c_{0}$ at $S=3, M=10$.

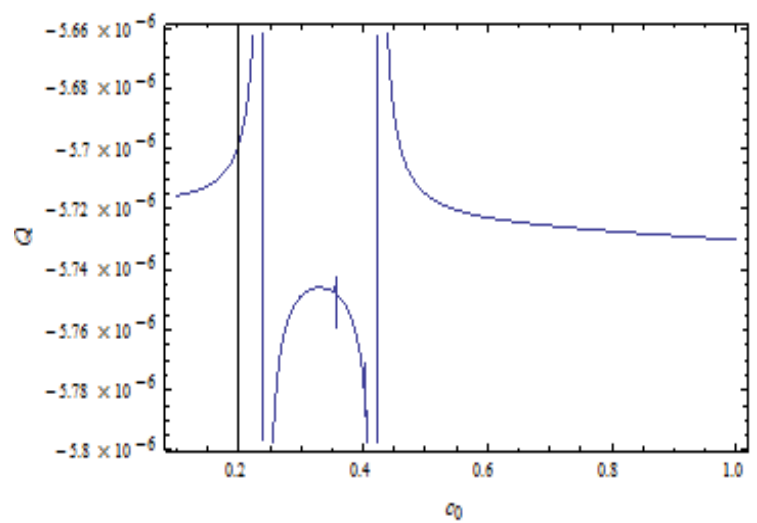

Figure 22b: Volumetric flow rate $Q$ with $c_{0}$ at $S=3, M=30$.

At: $L=0.2, h=0.5, s=3, \delta=0.4, \beta_{v}=0.1, k_{m}=0.25, k_{p}^{*}=3$

with $y$ direction for different values of porous paramete $k_{p}^{*}$. Figures $9 \mathrm{c}$ and $9 \mathrm{~d}$ show that as $k_{p}^{*}$ increases the microrotation $y$ component $A$ increases. The relation between $A$ and coupling parameter $c_{0}$ is shown in Figures 10a, 10b and 10c. By observing Figure 10c, the micro rotation $y$ component $A$ is increasing as the coupling parameter $c_{0}$ is increasing. Figures $10 \mathrm{a}$ and $10 \mathrm{~b}$ indicate $A$ with $y$ direction for different values of $c_{0}$. Figures $11 \mathrm{a}, 11 \mathrm{~b}$ and $11 \mathrm{c}$ illustrate $A$ with $y$ direction for different values of couple stress parameter $s$, the couple stress parameter $k_{p}^{*}$ increases and the microrotation $y$ component $A$ increases. From

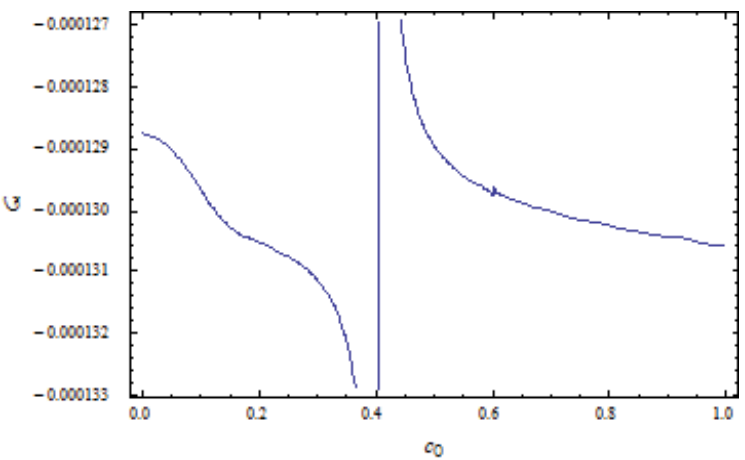

Figure 22c: Relation between volumetric flow rate $Q$ and $C_{0}$ At: $L=0.2, h=0.8, s=3, M=10, \delta=0.4, \beta_{v}=0.1, k_{m}=0.25, k_{p}^{*}=3$

Figures $12 \mathrm{a}$ and $12 \mathrm{~b}$ we show that $A$ decreases with Kundsen number $k_{m}$ increases.

Figures 13-18 illustrate the variation of the microrotation $z$ component $B$ with different parameters. The flow pattern of $B$ is shown in Figures 13a and 13c for different values of $M$. The contour lines of $B$ are shown in Figure $13 \mathrm{~b}$ at $\mathrm{M}=10$ and Figure $13 \mathrm{~d}$ at $M=10$. From Figures $14 \mathrm{a}, 14 \mathrm{~b}$ and $14 \mathrm{c} B$ is increasing as $M$ increasing. The microrotation $z$ component $B$ decreasing as the porous parameter $k_{p}^{*}$ increasing, this is shown in Figures $15 \mathrm{a}, 15 \mathrm{~b}, 15 \mathrm{c}$ and $15 \mathrm{~d}$. Figures $16 \mathrm{a}, 16 \mathrm{~b}$ and $16 \mathrm{c}$ depict the effect of coupling parameter $c_{0}$ on $B$, as $c_{0}$ increases the microrotation $z$ component $B$ increases. From Figures $17 \mathrm{a}, 17 \mathrm{~b}$ and $17 \mathrm{c}$, it can be observed that $B$ is decreasing with couple stress parameter $s$ increasing. When Kundsen number $k_{m}$ increases the microrotation $z$ component $B$ will be decreases this is shown in Figures $18 \mathrm{a}$ and $18 \mathrm{~b}$.

Volumetric flow rate is given by equation (37) and it is numerically calculated and is shown in the form of Figures 19-22. From these we observe that volumetric flow rate $Q$ increases when the couple stress parameter $S$ increases, the magnetic parameter $M$ increases and the Kundsen number $k_{m}$ increases. But volumetric flow rate $Q$ decreases as coupling parameter $c_{0}$ decreases and porous parameter $k_{p}^{*}$ decreases.

\section{Conclusions}

The present results make us to understand, numerically as well as physically, the influence of $M, k_{p}^{*}, c_{0}, s$ and $k_{m}$ on the slip flow of micropolar fluid through a porous medium in a rectangular microchannel effected by a uniform magnetic field.

(1) The velocity $u$ and microrotation component $A$ are decreasing with the increase in the value of $M$ while the microrotation component $B$ and volumetric flow rate $Q$ are increasing with the increase in $M$.

(2) The increasing of porous parameter $k_{p}^{*}$ acts to increase $u$ and $A$, decrease $B$ and $Q$.

(3) As the coupling parameter $c_{0}$ is increasing the velocity $u$ and $A$ are increasing but volumetric flow rate decreasing while $B$ decreases and increases in different regions.

(4) Increasing the couple stress parameter $s$ and hence decreasing in $u$ and $B$ but increasing in $A$ and $Q$.

(5) The velocity $u$ and volumetric flow rate $Q$ are increasing with the increase in the Kundsen number $k_{m}$, but the microrotation component $A$ and $B$ are decreasing when $k_{m}$ increases. 
Citation: Agoor BM (2018) Slip Flow in Porous Medium of Micropolar Fluid in a Rectangular Microchannel under the Effect of a Magnetic Field. J Appl Mech Eng 7: 298. doi:10.4172/2168-9873.1000298

Page 14 of 14

\section{Acknowledgment}

The author wishes to express his sincere thanks to Professor Nabil T. Eldabe, Faculty of Education, Department of Mathematics, Ain Shams University, Cairo Egypt, for his valuable suggestions and moral support. The author is indebted to him to review the manuscript and for helpful comments.

\section{References}

1. Eringen AC (1966) Theory of micropolar fluids. J Math Mech 16: 1-8.

2. Hogan HA, Henriksen M (1989) An evaluation of a micropolar model for blood flow through an idealized stenosis. J Biomech 22: 211-218.

3. Sparrow EM, Lin SH, Tslundgren S (1964) Flow development in the hydrodynamic entrance region of tubes and ducts. Phys Fluids 7: $338-347$.

4. Comini G, Del Giudice S, Strada M (1980) Finite element analysis of laminar flow in the entrance region of ducts. Int $\mathrm{J}$ for Numerical Methods in Engineering 5: 507-517.

5. Chien $F(1965)$ Unsteady laminar incompressible flow through rectangular duct. ZAMP 169: 351-360.

6. Gupta PC, Sarma RG (1982) Unsteady flow of viscous fluids in a porous rectangular tube. Bull. Cal Math Soc 74: 353-357.

7. Erdogan EM, Imrak EC (2004) Effects of the side walls on the unsteady flow of a second grade fluid in a duct of uniform cross section. Int $\mathrm{J}$ Nonlinear Mechanics 39: 1379-1384.

8. Murthy RJV, Sai KS, Bahali NK (2011) Steady flow of micropolar fluid in a rectangular channel under transverse magnetic field with suction. AIP Advances.
9. Andersson HI, Trondheim S (2002) Slip flow past a stretching surface. Acta Mechanica 158:121-125.

10. Misra JC, Shit GC (2007) Role of slip velocity in blood flow through stenosed arteries: A Non-Newtonian model. J Mech Med Biol 07: 337.

11. Lok YY, Pop L, Ingham DB (2010) Oblique stagnation slip flow of a micropolar fluid. Meccanica 45: 187-198.

12. Adanhounme V, De Paule Codo F, Adomou A (2012) Solving the NavierStokes flow equations of micropolar fluids by a domain decomposition method. Bulletin of Society for Mathematical Services and Standards 1: 35-42.

13. Shangjun Y, Zhu K, Wang W (2006) Laminar flow of micropolar fluid in rectangular Microchannels. Acta Mechanica Sinica 22: 403-408.

14. Vimala $P$, Blessie $P$, Omega $F(2015)$ Solution of micropolar fluid flow through porous channels a differential transform approach. Applied Mathematical Science 9: 3291-3302.

15. Misra JC, Chandra S, Herwing H (2015) Flow of a micropolar fluid in a microchannel under the action of an alternating electric field: Estimates of flow in bio-fluidic devices. J Hydrodynamics Ser. B 27: 350-358.

16. Eringen AC (1964) Simple micro fluids. Int J Eng Sci 2: 205 -217.

17. Eckert EGR, Drake RM (1972) Analysis of heat and mass transfer. Mc GrawHill New York, USA. 2: 467-486.

18. Goniak R, Duffa G (1995) Corrective term in wall slip equations for Knudsen layer. J Thermophys 9: 383-384. 